\title{
Idempotents in the Neighbourhood of Patterson-Wiedemann Functions having Walsh Spectra Zeros*
}

\author{
Sumanta Sarkar and Subhamoy Maitra \\ Applied Statistics Unit, Indian Statistical Institute \\ 203 B T Road, Kolkata 700 108, India \\ Email: \{sumanta_r, subho\}@isical.ac.in
}

\begin{abstract}
In this paper we study the neighbourhood of 15 -variable Patterson-Wiedemann (PW) functions, i.e., the functions that differ by a small Hamming distance from the PW functions in terms of truth table representation. We exploit the idempotent structure of the PW functions and interpret them as Rotation Symmetric Boolean Functions (RSBFs). We present techniques to modify these RSBFs to introduce zeros in the Walsh spectra of the modified functions with minimum reduction in nonlinearity. Our technique demonstrates 15 -variable balanced and 1-resilient functions with currently best known nonlinearities 16272 and 16264 respectively. In the process, we find functions for which the autocorrelation spectra and algebraic immunity parameters are best known till date.
\end{abstract}

keywords: Algebraic Immunity, Autocorrelation, Balancedness, Nonlinearity, Rotation Symmetric Boolean Functions, Resiliency.

\section{Introduction}

In [25], Patterson and Wiedemann presented Boolean functions on 15-variables with nonlinearity strictly greater than the bent concatenation bound. After more than two decades, in [15], 9 -variable functions having nonlinearity exceeding the bent concatenation bound have been demonstrated. Most interestingly, both these constructions rely on the idempotent structure of the Boolean functions. Under the interpretation that a Boolean function is a mapping $f: G F\left(2^{n}\right) \rightarrow G F(2)$, the functions presented in [13,15,25] are such that $f\left(x^{2}\right)=f(x)$ for any $x \in G F\left(2^{n}\right)$. These functions were studied in [9-11] and referred as idempotents. By fixing any irreducible polynomial of degree $n$ over $\mathrm{GF}(2)$, one may interpret the mapping $f: G F\left(2^{n}\right) \rightarrow G F(2)$ as $f:\{0,1\}^{n} \rightarrow\{0,1\}$. One can use this interpretation to get a Rotation Symmetric Boolean Function (RSBF) from an idempotent by choosing a primitive polynomial of degree $n$ and a normal basis [9]. The RSBFs are studied in great detail recently and it has been found that this sub class of Boolean functions is extremely rich in terms of cryptographic and combinatorial properties $[4,6,12-14,20,21,27,35,36]$. Motivated by these results, we concentrate on $\mathrm{PW}$ functions in this paper and exploit the rotation symmetric structure of such functions to get best known nonlinearity results in terms of balanced and 1-resilient functions.

High nonlinearity of a Boolean function is important when it is used as a building block in any cryptographic system. On the other hand nonlinearity of a Boolean function is directly

${ }^{*}$ This is a revised and extended (Section 4 is a new addition) version of the paper that has been presented in WCC 2007, International Workshop on Coding and Cryptography, April 16-20, 2007, Versailles (France). 
related to the covering radius of first order Reed-Muller codes. It is well known that the maximum possible nonlinearity of an $n$-variable Boolean function is $2^{n-1}-2^{\frac{n}{2}-1}$ for $n$ even [7, $29]$ and functions with this nonlinearity are called bent functions. The bound $2^{n-1}-\left\lceil 2^{\frac{n}{2}-1}\right\rceil$ is in general not known to be achieved when $n$ is odd. For odd $n$, one can easily get (balanced) Boolean functions having nonlinearity $2^{n-1}-2^{\frac{n-1}{2}}$ by suitably concatenating two bent functions on $(n-1)$ variables. That is the reason the nonlinearity value $2^{n-1}-2^{\frac{n-1}{2}}$ for odd $n$ is called the bent concatenation bound. For odd $n \leq 7$, the maximum nonlinearity of $n$-variable functions is $2^{n-1}-2^{\frac{n-1}{2}}[1,23]$ and for odd $n>7$, the maximum nonlinearity can exceed this bound $[13,15,25]$.

Since balancedness is a useful cryptographic property for a Boolean function, the question of getting balanced Boolean function with high nonlinearity is an important issue. Further it is also combinatorially very interesting. As the bent functions are not balanced, the maximum nonlinearity for $n$-variable balanced functions for even $n$ must be less than $2^{n-1}-2^{\frac{n}{2}-1}$. Denote the maximum nonlinearity for any balanced Boolean function on $b$-variables by $n l b(b)$. Dobbertin conjectured in [8] that for $n$ even, $n l b(n) \ngtr 2^{n-1}-2^{\frac{n}{2}}+n l b\left(\frac{n}{2}\right)$. This conjecture still remains unsettled.

For odd $n$, the challenge is to get balanced Boolean functions having nonlinearity greater than the bent concatenation bound. The first attempt in this direction was in [34], where 15-variable PW functions were used as a black box to construct balanced functions on odd number of input variables $(\geq 29)$ having nonlinearity greater than the bent concatenation bound. Later, in $[19,30]$, the truth tables of the PW functions were modified to get 15 -variable balanced functions having nonlinearity 16262 and that shows the existence of balanced Boolean functions exceeding the bent concatenation bound for odd number of input variables greater than or equal to 15 .

Before explaining our contribution in detail, we first present some preliminaries.

\subsection{Basics of Boolean functions}

An $n$-variable Boolean function $f$ is a mapping $f: G F\left(2^{n}\right) \rightarrow G F(2)$. Another representation of a Boolean function $f$ is a mapping $f:\{0,1\}^{n} \rightarrow\{0,1\}$. This representation is called the truth table representation. Using any basis of $G F\left(2^{n}\right)$, we can express each $x \in G F\left(2^{n}\right)$ as an $n$-tuple $\left(x_{1} x_{2} \ldots x_{n}\right), x_{i} \in G F(2), i=1, \ldots, n$. Thus we can draw the truth table representation from the former representation.

We now concentrate on the truth table representation of a Boolean function which is a $2^{n}$ length binary string

$$
f=[f(0,0, \cdots, 0), f(1,0, \cdots, 0), f(0,1, \cdots, 0), \ldots, f(1,1, \cdots, 1)] .
$$

The Hamming weight of a binary string $T$ is the number of 1's in $T$, denoted by $w t(T)$. An $n$-variable function $f$ is said to be balanced if its truth table contains an equal number of 0 's and 1's, i.e., $w t(f)=2^{n-1}$. Also, the Hamming distance between two equidimensional binary strings $T_{1}$ and $T_{2}$ is defined by $d\left(T_{1}, T_{2}\right)=w t\left(T_{1} \oplus T_{2}\right)$, where $\oplus$ denotes the addition over $G F(2)$.

An $n$-variable Boolean function $f\left(x_{1}, \ldots, x_{n}\right)$ can be considered to be a multivariate polynomial over $G F(2)$. This polynomial can be expressed as a sum of products representation of all distinct $k$-th order products $(0 \leq k \leq n)$ of the variables. More precisely, $f\left(x_{1}, \ldots, x_{n}\right)$ can be written as

$$
a_{0} \oplus \bigoplus_{1 \leq i \leq n} a_{i} x_{i} \oplus \bigoplus_{1 \leq i<j \leq n} a_{i j} x_{i} x_{j} \oplus \ldots \oplus a_{12 \ldots n} x_{1} x_{2} \ldots x_{n}
$$


where the coefficients $a_{0}, a_{i}, a_{i j}, \ldots, a_{12 \ldots n} \in\{0,1\}$. This representation of $f$ is called the algebraic normal form (ANF) of $f$. The number of variables in the highest order product term with nonzero coefficient is called the algebraic degree, or simply the degree of $f$ and denoted by $\operatorname{deg}(f)$.

Functions of degree at most one are called affine functions. An affine function with constant term equal to zero is called a linear function. The set of all $n$-variable affine (respectively linear) functions is denoted by $A(n)$ (respectively $L(n)$ ). The nonlinearity of an $n$-variable function $f$ is defined as

$$
n l(f)=\min _{g \in A(n)}(d(f, g)),
$$

i.e., the minimum distance from the set of all $n$-variable affine functions.

Let $x=\left(x_{1}, \ldots, x_{n}\right)$ and $\omega=\left(\omega_{1}, \ldots, \omega_{n}\right)$ both belong to $\{0,1\}^{n}$ and $x \cdot \omega=x_{1} \omega_{1} \oplus \ldots \oplus$ $x_{n} \omega_{n}$. Let $f(x)$ be a Boolean function on $n$ variables. Then the Walsh transform of $f(x)$ is an integer valued function over $\{0,1\}^{n}$ which is defined as

$$
W_{f}(\omega)=\sum_{x \in\{0,1\}^{n}}(-1)^{f(x) \oplus x \cdot \omega} .
$$

The Walsh spectrum of $f$ is the multiset $\left\{W_{f}(\omega) \mid \omega \in\{0,1\}^{n}\right\}$. In terms of Walsh spectrum, the nonlinearity of $f$ is given by

$$
n l(f)=2^{n-1}-\frac{1}{2} \max _{\omega \in\{0,1\}^{n}}\left|W_{f}(\omega)\right| .
$$

In [37], an important characterization of correlation immune functions has been presented, which we use as the definition here. A function $f\left(x_{1}, \ldots, x_{n}\right)$ is $m$-th order correlation immune (respectively $m$-resilient) iff its Walsh spectrum satisfies $W_{f}(\omega)=0$, for $1 \leq w t(\omega) \leq m$ (respectively $0 \leq w t(\omega) \leq m$ ).

Autocorrelation properties are also cryptographically important $[28,38]$ for a Boolean function $f$. Let $\beta \in\{0,1\}^{n}$. The autocorrelation value of the Boolean function $f$ with respect to the vector $\beta$ is $\Delta_{f}(\beta)=\sum_{x \in\{0,1\}^{n}}(-1)^{f(x) \oplus f(x \oplus \beta)}$. Further we denote

$$
\Delta_{f}=\max _{\beta \in\{0,1\}^{n}, \beta \neq(0, \ldots, 0)}\left|\Delta_{f}(\beta)\right|
$$

and $\Delta_{f}$ is called the absolute indicator. $f$ is said to satisfy $\operatorname{PC}(k)$, if $\Delta_{f}(\beta)=0$ for $1 \leq$ $w t(\beta) \leq k$.

Recently algebraic attack has received a lot of attention (see [2,3,22] and the references in these paper) in studying the security of the ciphers. One necessary condition to resist this attack is that the Boolean function used in the cryptosystem should have good algebraic immunity. An $n$-variable Boolean function $g$ is called an annihilator of an $n$-variable Boolean function $f$ if $f g=0$. We denote the set of all nonzero annihilators of $f$ by $A N(f)$. Then algebraic immunity of $f$, denoted by $\mathcal{A I}_{n}(f)$, is defined [22] as the degree of the minimum degree annihilator among all the annihilators of $f$ and $1+f$, i.e., $\mathcal{A I}_{n}(f)=\min \{\operatorname{deg}(g): g \neq$ $0, g \in A N(f) \cup A N(1+f)\}$. It is known $[3,22]$ that $\mathcal{A I}_{n}(f) \leq\left\lceil\frac{n}{2}\right\rceil$.

\subsection{Rotation Symmetric Boolean Function (RSBF)}

Let $x_{i} \in\{0,1\}$ for $1 \leq i \leq n$. For some integer $k \geq 0$ we define $\rho_{n}^{k}\left(x_{i}\right)$ as $\rho_{n}^{k}\left(x_{i}\right)=x_{i+k \bmod n}$, with the exception that when $i+k \equiv 0 \bmod n$, then we will assign $i+k \bmod n$ by $n$ instead of 0 . This is to cope up with the input variable indices $1, \ldots, n$ for $x_{1}, \ldots, x_{n}$. Let 
$\left(x_{1}, x_{2}, \ldots, x_{n-1}, x_{n}\right) \in\{0,1\}^{n}$. Then we extend the definition as $\rho_{n}^{k}\left(x_{1}, x_{2}, \ldots, x_{n-1}, x_{n}\right)=$ $\left(\rho_{n}^{k}\left(x_{1}\right), \rho_{n}^{k}\left(x_{2}\right), \ldots, \rho_{n}^{k}\left(x_{n-1}\right), \rho_{n}^{k}\left(x_{n}\right)\right)$. Hence, $\rho_{n}^{k}$ acts as $k$-cyclic rotation on an $n$-bit vector.

A Boolean function $f$ is called rotation symmetric if for each input $\left(x_{1}, \ldots, x_{n}\right) \in\{0,1\}^{n}$,

$$
f\left(\rho_{n}^{k}\left(x_{1}, \ldots, x_{n}\right)\right)=f\left(x_{1}, \ldots, x_{n}\right) \text { for } 1 \leq k \leq n-1 .
$$

That is, the rotation symmetric Boolean functions are invariant under cyclic rotations of inputs. The inputs of a rotation symmetric Boolean function can be divided into orbits so that each orbit consists of all cyclic shifts of one input. An orbit generated by $\left(x_{1}, x_{2}, \ldots, x_{n}\right)$ is

$$
G_{n}\left(x_{1}, x_{2}, \ldots, x_{n}\right)=\left\{\rho_{n}^{k}\left(x_{1}, x_{2}, \ldots, x_{n}\right) \mid 1 \leq k \leq n\right\}
$$

and the number of such orbits is denoted by $g_{n}$. Thus the total number of distinct $n$-variable RSBFs is $2^{g_{n}}$. Let $\phi$ be Euler's $p h i$-function, then it can be shown by Burnside's lemma that (see also [35])

$$
g_{n}=\frac{1}{n} \sum_{k \mid n} \phi(k) 2^{\frac{n}{k}}
$$

An orbit is completely determined by its representative element $\Lambda_{n, i}$, which is the lexicographically first element belonging to the orbit [36]. These representative elements are again arranged lexicographically as $\Lambda_{n, 0}, \ldots, \Lambda_{n, g_{n}-1}$. Thus an $n$-variable RSBF $f$ can be represented by the $g_{n}$ length string $\left[f\left(\Lambda_{n, 0}\right), \ldots, f\left(\Lambda_{n, g_{n}-1}\right)\right]$.

In [36] it was shown that the Walsh spectrum of an RSBF $f$ takes the same value for all elements belonging to the same orbit, i.e., $W_{f}(u)=W_{f}(v)$ if $u \in G_{n}(v)$. Therefore the Walsh spectrum of $f$ can be represented by the $g_{n}$ length vector $\left(w a_{f}[0], \ldots, w a_{f}\left[g_{n}-1\right]\right)$, where $w a_{f}[j]=W_{f}\left(\Lambda_{n, j}\right)$. In analyzing the Walsh spectrum of an RSBF, the ${ }_{n} \mathcal{A}$ matrix has been introduced [36]. The matrix ${ }_{n} \mathcal{A}=\left({ }_{n} \mathcal{A}_{i, j}\right)_{g_{n} \times g_{n}}$ is defined as

$$
{ }_{n} \mathcal{A}_{i, j}=\sum_{x \in G_{n}\left(\Lambda_{n, i}\right)}(-1)^{x \cdot \Lambda_{n, j}}
$$

for an $n$-variable RSBF. Using this $g_{n} \times g_{n}$ matrix, the Walsh spectrum for an RSBF can be calculated as

$$
W_{f}\left(\Lambda_{n, j}\right)=\sum_{i=0}^{g_{n}-1}(-1)^{f\left(\Lambda_{n, i}\right)}{ }_{n} \mathcal{A}_{i, j} .
$$

The operation of multiplying by 2 divides the integers $\bmod \left(2^{n}-1\right)$ into different sets called 2 -cyclotomic cosets $\bmod \left(2^{n}-1\right)$. The 2 -cyclotomic coset containing $s$ consists of the elements $\left\{s, 2 s, 2^{2} s, \ldots, 2^{d_{s}-1} s\right\}$ where $d_{s}$ is the smallest positive integer such that $2^{d_{s}} \cdot s \equiv s \bmod \left(2^{n}-1\right)$. The term $d_{s}$ is called the length of the cyclotomic coset $\bmod \left(2^{n}-1\right)$. One may note that there are $\left(g_{n}-1\right)$ many cyclotomic cosets.

\subsection{Equivalence between RSBF and Idempotent}

Let us consider a Boolean function $f: G F\left(2^{n}\right) \rightarrow G F(2)$. A Boolean function $f$ is called idempotent [9] iff $f(\gamma)=f\left(\gamma^{2}\right)$, for any $\gamma \in G F\left(2^{n}\right)$. Given a primitive element $\theta \in G F\left(2^{n}\right)$, an idempotent function will have the same value corresponding to all elements $\theta^{i}$ where $i$ belongs to the same 2-cyclotomic coset, say $\left\{s, 2 s, 2^{2} s, \ldots, 2^{d_{s}-1} s\right\}$.

We fix a primitive polynomial $P(X)$ of degree $n$ over $\mathrm{GF}(2)$ and let $\theta$ be a root of $P(X)$. Let us consider a normal basis $\left\{\theta^{t}, \theta^{2 t}, \theta^{2^{2} t} \ldots, \theta^{2^{n-1} t}\right\}$ of $G F\left(2^{n}\right)$. We represent $\theta^{t}, \theta^{2 t}, \theta^{2^{2} t}, \ldots, \theta^{2^{n-1} t}$ by the $n$-bit binary vectors $(1,0, \ldots, 0),(0,1, \ldots, 0), \ldots,(0,0, \ldots, 1)$ (the 
order is $\left.\left(x_{1}, x_{2}, \ldots, x_{n}\right)\right)$ respectively. Then all the elements $\theta^{j}$ can be expressed as an $n$-bit binary vector with respect to the normal basis. Once the $n$-bit vector is decided, this is basically the assignment to the inputs of the Boolean function and we can refer back to the standard truth table (considering Boolean function as a mapping $\{0,1\}^{n} \rightarrow\{0,1\}$ ) to get the value of the function corresponding to the input pattern. In this representation all the $n$-bit binary vectors corresponding to the elements $\left\{\theta^{s}, \theta^{2 s}, \theta^{2^{2} s} \ldots, \theta^{2^{d_{s}-1} s}\right\}$ will be cyclic rotation of each other [9]. That means the elements $\theta^{i}$ where $i$ runs over a 2-cyclotomic coset, form an orbit and as the idempotent $f$ has the same value corresponding to all these $\theta^{i}$ 's, $f$ will have the same output in its truth table for all the elements in the orbit; i.e., in terms of truth table representation, $f$ becomes an $\mathrm{RSBF}$.

\subsection{Contribution of this paper}

Balancedness is an important property of a Boolean function from a cryptographic as well as a combinatorial point of view. A challenging question in this direction is to get balanced Boolean functions with high nonlinearity. One natural attempt for the 15-variable case is to use or modify the PW functions (which do not contain any zero in their Walsh spectra) to get balancedness, keeping in mind that the nonlinearity should not decrease much due to the modification.

The 15-variable PW functions were used as a black box in [34] to construct balanced functions on an odd number of input variables $(\geq 29)$ having nonlinearity greater than the bent concatenation bound. However, the internal structure of the PW functions was not studied in [34]. In $[19,30]$ the internal structure of the PW functions has been modified to get improved results upon [34] in terms of nonlinearity for balanced functions on odd number $(\geq 15)$ of input variables. The idea of $[19,30]$ was as follows.

Take $n=15$. Consider the truth table of a PW function $f$ as a mapping from $\{0,1\}^{n} \rightarrow$ $\{0,1\}$. One can easily check that there are 3255 many points $\omega \in\{0,1\}^{n}$ where the value of the Walsh spectrum $W_{f}(\omega)=40$. Now consider a function $g=f \oplus \omega \cdot x$. Clearly $W_{g}(0)=40$ and one needs to toggle 20 output bits from 0 to 1 to achieve balancedness. The idea of $[19,30]$ was to divide the $2^{n}$-bit long truth table of $g$ in 20 (almost) equal contiguous parts and selecting a random 0 bit from each part and toggle that to 1 . Thus the modified function from $g$ becomes balanced and in some of the cases the reduction in nonlinearity was less than 20. That provided the nonlinearity greater than the bent concatenation bound. Though the simple method provided nice results, it was only a heuristic and the idempotent structure of the PW functions was not exploited at all. In this paper we look at the idempotent structure of the PW functions and get better results over $[19,30]$. In this direction, first we interpret the PW functions as RSBFs in order to take the advantage of the matrix ${ }_{n} \mathcal{A}$ associated with the Walsh transform of the functions. Then studying the distribution of the Walsh spectra values, we modify the PW functions to obtain new functions having high nonlinearity. Let us list the results we achieve in this paper that were not known earlier.

1. In Section 2.1, by modifying the two available PW functions from [25] we find 316 many RSBFs (idempotents) each having nonlinearity 16264 and 15 Walsh spectrum zeros. All these 316 functions have a different distribution in the Walsh spectra and they are not affinely equivalent among themselves. These functions can be transformed to balanced functions and this nonlinearity 16264 is better than the nonlinearity 16262 presented in $[19,30]$. Once more we like to point out that this study is a more disciplined one in terms of exploiting the structure of the idempotents rather than the simple heuristic presented in $[19,30]$. Some of these functions have the maximum absolute value in the 
autocorrelation spectra as low as 192, which is better than 216 as presented in $[19,30]$. Further we find functions having maximum possible algebraic immunity 8 where the maximum absolute value in the autocorrelation spectra is as low as 200 . This is the first time a function on an odd number of variables having maximum possible algebraic immunity with nonlinearity greater than the bent concatenation bound is demonstrated. In [5], the functions of $[19,30]$ have been studied for their algebraic immunity and the value found was 7 , which is not the maximum possible.

2. In Section 3, we further modify some of the 316 RSBFs (reported in Subsection 2.1) by toggling the outputs corresponding to two input points and could achieve balanced functions with nonlinearity 16266, algebraic immunity 8 and maximum absolute value in the autocorrelation spectra 208. Again, this is not done by randomly modifying two output points, but following a specific strategy examining the Walsh spectra of the functions.

After that we searched by modifying three orbits of size 15 and one orbit of size 1 . By that manner we could find 15-variable balanced function having nonlinearity 16272 . The nonlinearity presented here is the best known for 15-variable balanced functions and it provides the construction of $n$-variable ( $n \geq 15$ and odd) balanced functions having nonlinearity $2^{n-1}-2^{\frac{n-1}{2}}+16 \times 2^{\frac{n-15}{2}}$.

3. Each of the 15-variable functions presented in Section 2.1 and Section 3 (having nonlinearity either 16264 or 16266 ) has 15 many Walsh spectrum zeros. Unfortunately one needs at least 16 many zeros to have an attempt to get a 1-resilient function by the method of linear transformation on input variables. Thus we target some of the functions with certain distribution in the Walsh spectra having nonlinearity 16264 and modify each of them to increase the number of Walsh spectrum zeros keeping the rotation symmetric structure unchanged. We concentrate on the points where the Walsh spectrum values are close to zero and modify the function accordingly so that the values at those points can be changed to zero increasing the overall number of zeros in the Walsh spectrum. This technique has the risk that the nonlinearity will be reduced further, but we managed to control the reduction so that the resulting nonlinearity remains greater than the bent concatenation bound. In Section 4, we could modify the functions having nonlinearity 16264 from Section 2.1 to get functions with nonlinearity 16260 or 16264, each having 30 or more Walsh spectrum zeros. For example, we could get a function with nonlinearity 16264 with 135 many zeros in the Walsh spectrum that has then been suitably modified to 1-resilient function by linear transformation on input variables [18,24]. This shows that it is possible to construct 1-resilient functions having nonlinearity greater than the bent concatenation bound for $(15+2 i)$ variables $(i \geq 0)$. The maximum absolute autocorrelation value of this function is 232 . This shows for the first time the existence of a 1-resilient function exceeding the bent concatenation bound in nonlinearity with the maximum absolute value in the autocorrelation spectrum less than $2^{\frac{15+1}{2}}$.

Earlier 1-resilient 15-variable functions having nonlinearity greater than the bent concatenation bound were known for odd number of variables greater than or equal to 41 [30,32]. In [30,32], the 15-variable PW functions have been used in the construction of resilient functions but modification of the internal structure was not attempted to get resiliency. Thus 15-variable 1-resilient functions with nonlinearity more than the bent concatenation bound could not be identified in [30,32]. Our work is based on the modification of internal structure of PW functions and it shows the construction of 1-resilient functions on $n$ variables having nonlinearity 
strictly greater than $2^{n-1}-2^{\frac{n-1}{2}}$ for $n \geq 15$ and odd. Thus the gap from 15 to 39 variables is resolved by our work in terms of getting 1-resilient functions having nonlinearity $>2^{n-1}-2^{\frac{n-1}{2}}$. Further our nonlinearity is better than what was presented for the 41-variable case in $[30,32]$.

A more general study of Boolean functions invariant under the action of some finite groups has been presented in [17] that demonstrated 15-variable functions with nonlinearity greater than the bent concatenation bound (but not exceeding the nonlinearity reported in [25]). Our study can be considered as looking into a particular case where the functions are invariant under the action of the group of Frobenius automorphisms.

\section{Studying the Walsh Spectrum of PW functions as RSBF}

We first present the construction of RSBFs from the two PW functions on $(n=15)$-variables given in [25]. Each of these functions is idempotent when we consider them as a mapping from $G F\left(2^{n}\right)$ to $G F(2)$. Let $f_{P W}$ denotes one such function.

\section{Construction 1}

Take $n=15$

Consider a $P W$ function $f_{P W}$ on n-variables.

Take the primitive polynomial $P(X)=X^{15}+X+1$ over $G F(2)$.

Consider a root $\alpha$ of $P(X)$.

Take the normal basis $\mathcal{N}=\left\{\alpha^{\left(2^{i} \cdot 29\right) \bmod \left(2^{15}-1\right)}: i=0, \ldots, 14\right\}$.

Represent each $x \in G F\left(2^{n}\right)$ as an n-bit binary vector with respect to $\mathcal{N}$.

Denote the corresponding mapping $\{0,1\}^{n} \rightarrow\{0,1\}$ by $f$.

$f$ is an $R S B F$ with $n l(f)=2^{n-1}-2^{\frac{n-1}{2}}+20=16276$.

In the rest of the paper we will consider $f$ as the RSBF obtained from a PW function using Construction 1. We get two distinct (the first one is of algebraic degree 8 and the second one is of algebraic degree 9) RSBFs up to affine equivalence from Construction 1. Each of them are of nonlinearity 16276 and the distribution of Walsh spectra of both the functions are the same (presented in Table 1).

For $n=15$, the number of orbits is $g_{n}=2192$, out of them there are 2182 orbits of size 15 , 6 orbits of size 5,2 orbits of size 3 and 2 orbits of size 1 .

Table 1: Distribution of Walsh spectrum for 15-variable PW function.

\begin{tabular}{|c|c|c|c|}
\hline $\begin{array}{c}\text { Weight } \\
w\end{array}$ & $\begin{array}{c}\text { Number of Vectors } \\
\text { (number of input points) }\end{array}$ & $\begin{array}{c}\text { Walsh Spectra Value } \\
2^{15}-2 w\end{array}$ & $\begin{array}{c}\text { How it comes } \\
\text { (\# of orbits of size 15, 5, 3, 1) }\end{array}$ \\
\hline 16492 & 13021 & -216 & $868,0,0,1$ \\
\hline 16428 & 217 & -88 & $12,6,2,1$ \\
\hline 16364 & 3255 & 40 & $217,0,0,0$ \\
\hline 16300 & 16275 & 168 & $1085,0,0,0$ \\
\hline
\end{tabular}

We are interested in modifying each of the PW functions such that we can get zeros in the Walsh spectrum with minimum number of toggles at the output bits. A random strategy has been presented in [30] that we have briefly explained in the previous section. Here our motivation is to toggle the outputs of $f$ corresponding to one or more orbits. It means that after the modification, the function will remain RSBF. 


\subsection{Modification with respect to one orbit of size 15 and another of size 5}

We first start with a theoretical result.

Theorem 1 Refer to the function $f$ as in Construction 1. Let $G_{n}\left(\Lambda_{n, j}\right)$ be an orbit such that $W_{f}\left(\Lambda_{n, j}\right)=40$ and

$$
(-1)^{f\left(\Lambda_{n, q}\right)}{ }_{n} \mathcal{A}_{q, j}+(-1)^{f\left(\Lambda_{n, r}\right)}{ }_{n} \mathcal{A}_{r, j}=20,
$$

for some $q, r$, where $\Lambda_{n, q}$ is the representative element of an orbit of size 15 and $\Lambda_{n, r}$ is the representative element of an orbit of size 5. Construct

$$
\begin{aligned}
g(x) & =f(x) \text { for } x \in\{0,1\}^{n} \backslash\left(\left(G_{n}\left(\Lambda_{n, q}\right) \cup G_{n}\left(\Lambda_{n, r}\right)\right),\right. \\
& =1 \oplus f(x) \text { for } x \in G_{n}\left(\Lambda_{n, q}\right) \cup G_{n}\left(\Lambda_{n, r}\right) .
\end{aligned}
$$

Then $W_{g}\left(\Lambda_{n, j}\right)=0$.

Further, let $\Lambda_{n, s}$ be the representative elements such that $W_{f}\left(\Lambda_{n, s}\right)=-216$ as $s$ varies. If $(-1)^{f\left(\Lambda_{n, q}\right)}{ }_{n} \mathcal{A}_{q, s}+(-1)^{f\left(\Lambda_{n, r}\right)}{ }_{n} \mathcal{A}_{r, s}<20$ for all $s$, then $n l(g)>2^{n-1}-2^{\frac{n-1}{2}}$.

Proof: Since, $(-1)^{f\left(\Lambda_{n, q}\right)}{ }_{n} \mathcal{A}_{q, j}+(-1)^{f\left(\Lambda_{n, r}\right)}{ }_{n} \mathcal{A}_{r, j}=20$, and $g=1 \oplus f$ for the input points corresponding to the orbits represented by $\Lambda_{n, q}, \Lambda_{n, r}$, we have,

$$
(-1)^{g\left(\Lambda_{n, q}\right)}{ }_{n} \mathcal{A}_{q, j}+(-1)^{g\left(\Lambda_{n, r}\right)}{ }_{n} \mathcal{A}_{r, j}=-20 .
$$

Also since $W_{f}\left(\Lambda_{n, j}\right)=40$ and $(-1)^{f\left(\Lambda_{n, q}\right)}{ }_{n} \mathcal{A}_{q, j}+(-1)^{f\left(\Lambda_{n, r}\right)}{ }_{n} \mathcal{A}_{r, j}=20$, therefore we have $\sum_{i \notin\{q, r\}}(-1)^{f\left(\Lambda_{n, i}\right)}{ }_{n} \mathcal{A}_{i, j}=20$. Thus,

$$
W_{g}\left(\Lambda_{n, j}\right)=\left((-1)^{g\left(\Lambda_{n, q}\right)}{ }_{n} \mathcal{A}_{q, j}+(-1)^{g\left(\Lambda_{n, r}\right)}{ }_{n} \mathcal{A}_{r, j}\right)+\sum_{i \notin\{q, r\}}(-1)^{g\left(\Lambda_{n, i}\right)}{ }_{n} \mathcal{A}_{i, j}=-20+20=0 .
$$

This proves the first part of the theorem.

Now refer to Table 1 and note that for any $\omega$, such that $W_{f}(\omega)=-88,40,168,\left|W_{g}(\omega)\right| \leq$ $168+40=208$. Further, consider the points $\Lambda_{n, s}$ where the Walsh spectrum values of $f$ are maximum in absolute terms, i.e., referring to Table 1 , we have $W_{f}\left(\Lambda_{n, s}\right)=-216$ as $s$ varies. Let $(-1)^{f\left(\Lambda_{n, q)}\right.}{ }_{n} \mathcal{A}_{q, s}+(-1)^{f\left(\Lambda_{n, r}\right)}{ }_{n} \mathcal{A}_{r, s}=20-\delta_{s}$, where $\delta_{s}>0$. Thus,

$$
\begin{aligned}
W_{g}\left(\Lambda_{n, s}\right) & =(-1)^{g\left(\Lambda_{n, q}\right)}{ }_{n} \mathcal{A}_{q, s}+(-1)^{g\left(\Lambda_{n, r}\right)}{ }_{n} \mathcal{A}_{r, s}+\sum_{i \in\{q, r\}}(-1)^{g\left(\Lambda_{n, i}\right)}{ }_{n} \mathcal{A}_{i, s} \\
& =-\left((-1)^{f\left(\Lambda_{n, q}\right)}{ }_{n} \mathcal{A}_{q, s}+(-1)^{f\left(\Lambda_{n, r}\right)}{ }_{n} \mathcal{A}_{r, s}\right)+\sum_{i \notin\{q, r\}}(-1)^{f\left(\Lambda_{n, i}\right)}{ }_{n} \mathcal{A}_{i, s} \\
& =-20+\delta_{s}+\left(-216-20+\delta_{s}\right)=-256+2 \delta_{s} .
\end{aligned}
$$

Thus $n l(g)>2^{n-1}-2^{\frac{n-1}{2}}$.

Using the idea of the above theorem, we design an algorithm to get 15-variable RSBFs $g$ such that $n l(g)>2^{n-1}-2^{\frac{n-1}{2}}$ with $W_{g}(\omega)=0$ for some point $\omega$. There are 217 orbits (each of size 15) at which the Walsh spectrum value of $f$ is 40 . We take an orbit $G_{n}\left(\Lambda_{n, j}\right)$ such that $W_{f}\left(\Lambda_{n, j}\right)=40$. Next we choose one orbit $G_{n}\left(\Lambda_{n, q}\right)$ of size 15 and another orbit $G_{n}\left(\Lambda_{n, r}\right)$ of size 5 such that

$$
(-1)^{f\left(\Lambda_{n, q}\right)}{ }_{n} \mathcal{A}_{q, j}+(-1)^{f\left(\Lambda_{n, r}\right)}{ }_{n} \mathcal{A}_{r, j}=20 .
$$

Then by Theorem 1 , we have $W_{g}\left(\Lambda_{n, j}\right)=0$, i.e., $W_{g}(\omega)=0$ for each $\omega \in G_{n}\left(\Lambda_{n, j}\right)$. As $\left|G_{n}\left(\Lambda_{n, j}\right)\right|=15$, number of the zeros in the Walsh spectrum of $g$ will be 15 .

Now we present the actual algorithm.

\section{Algorithm 1}


$\operatorname{maxnl}=0$;

for each of the representative elements $\lambda$ such that $W_{f}(\lambda)=40$

for each pair of the representative elements $\delta, \gamma$ with $\left|G_{n}(\delta)\right|=15,\left|G_{n}(\gamma)\right|=5$

$$
\operatorname{sum}=(-1)^{f(\delta)} \sum_{x \in G_{n}(\delta)}(-1)^{x \cdot \lambda}+(-1)^{f(\gamma)} \sum_{x \in G_{n}(\gamma)}(-1)^{x \cdot \lambda}
$$

if $\operatorname{sum}=20$

$$
\begin{aligned}
& g(x)=f(x) \text { for } x \in\{0,1\}^{n} \backslash\left(G_{n}(\delta) \cup G_{n}(\gamma)\right) \\
& g(x)=1 \oplus f(x) \text { for } x \in G_{n}(\delta) \cup G_{n}(\gamma) \\
& \text { if } \operatorname{maxnl}<n l(g) \text {, then } \operatorname{maxnl}=n l(g) \\
& \quad \text { store } \delta, \gamma \text { and } n l(g) \text { in a file } \mathrm{F} ;
\end{aligned}
$$

for each $\delta, \gamma, n l(g)$ tuple in a file $\mathrm{F}$

$$
\text { if } n l(g)<\operatorname{maxnl}
$$

remove the tuple from file $\mathrm{F}$;

file $\mathrm{F}$ provides the RSBFs with nonlinearity maxnl with at least 15 Walsh zeros;

\section{Complexity of Algorithm 1}

We define the following sets.

$S_{1}=\left\{\Lambda_{n, d}: W_{f}\left(\Lambda_{n, d}\right)=40\right\}, S_{2}=\left\{\Lambda_{n, d}:\left|G_{n}\left(\Lambda_{n, d}\right)\right|=15\right\}$ and $S_{3}=\left\{\Lambda_{n, d}:\left|G_{n}\left(\Lambda_{n, d}\right)\right|=5\right\}$.

Then we need to check $\left|S_{1}\right| \times\left|S_{2}\right| \times\left|S_{3}\right|$, i.e., $217 \times 2182 \times 6<2^{22}$ many options. For each of the options, we need to calculate the nonlinearity of $g$, requiring $O\left(n 2^{n}\right)$ time using the Fast Walsh Transform which is around $2^{19}$. Thus the total time complexity is around $2^{41}$, which is negligible compared to any search in the space of 15-variable Boolean functions.

\section{Outcome of Algorithm 1}

Running Algorithm 1 we get 253 and 63 RSBFs $g$ respectively from degree 9 and degree $8 \mathrm{PW}$ functions with nonlinearity maxnl $=16264$ and for each of these functions the Walsh spectrum contains 15 many zeros which occur exactly at an orbit of size 15 . We further check these functions and find that they are all affinely non-equivalent as their Walsh distributions are different.

Refer to Appendix A to get the list of these 316 functions with nonlinearity 16264 . We studied these functions and the distribution of the functions $g$ with respect to $\Delta_{g}$ is given in the following table.

Table 2: Number of RSBFs $g$ with nonlinearity 16264 with corresponding $\Delta_{g}$ values.

\begin{tabular}{|c|c|c|c|c|c|c|c|c|c|c|}
\hline$\Delta_{g}$ & 192 & 200 & 208 & 216 & 224 & 232 & 240 & 248 & 256 & 280 \\
\hline Number of functions $g$ & 1 & 21 & 87 & 101 & 60 & 34 & 8 & 2 & 1 & 1 \\
\hline
\end{tabular}

Now consider $\omega \in\{0,1\}^{n}$ such that $W_{g}(\omega)=0$. Then it is clear that the function $g^{\prime}(x)=$ $g(x) \oplus \omega \cdot x$ will be balanced and $n l\left(g^{\prime}\right)=n l(g)=16264, \Delta_{g^{\prime}}=\Delta_{g}=192$. Thus we get balanced functions $g^{\prime}$ having better nonlinearity and autocorrelation values than presented in [30]. Note that, though $g$ is an RSBF, the rotational symmetric property may be lost in $g^{\prime}$.

In all the following examples of this paper, we express any element $x \in\{0,1\}^{n}$ as the $n$-bit binary vector $\left(x_{n}, x_{n-1}, \ldots, x_{1}\right)$, where $x_{n}$ is the most significant bit.

Example 1 Now we provide the exact specification of a function $g$ having nonlinearity 16264 and $\Delta_{g}=192$. First we construct the RSBF $f$ from the PW function of degree 8 by using 
Construction 1. Then we toggle the outputs of $f$ corresponding to the orbits represented by $(0,0,0,0,1,1,1,0,0,1,1,0,0,1,1)$ (of size 15) and $(0,0,0,0,1,0,0,0,0,1,0,0,0,0,1)$ (of size 5 ) to get $g$. The function $g$ has the Walsh spectrum values zero corresponding to the orbit represented by $(0,0,0,0,1,1,1,0,1,1,0,0,1,0,1)$ (of size 15). The algebraic degree of $g$ is 13 . If we consider $\omega=(0,0,0,0,1,1,1,0,1,1,0,0,1,0,1)$, then $g^{\prime}(x)=g(x) \oplus \omega \cdot x$ will be balanced. We have also noted that the algebraic immunity of $g^{\prime}$ is 7 , which is not the maximum possible.

Example 2 Next we present a function with the maximum possible algebraic immunity 8 . We take the RSBF $f$ obtained from the 8-degree PW function using Construction 1. Then we toggle the outputs of $f$ corresponding to the orbits represented respectively by the elements $(0,0,0,0,1,1,0,0,1,1,0,1,0,1,1)$ (of size 15) and $(0,1,0,1,1,0,1,0,1,1,0,1,0,1,1)$ (of size 5) to get $g$. This function has the Walsh spectrum values zero for the orbit represented by $(0,0,0,0,1,1,1,0,1,1,0,0,1,0,1)$ (of size 15$)$. For this function $g$, we have nonlinearity 16264, $\Delta_{g}=200$ and algebraic degree 13 . Let $\omega=(0,0,0,0,1,1,1,0,1,1,0,0,1,0,1)$, then $g^{\prime}(x)=$ $g(x) \oplus \omega \cdot x$ will be balanced and $g^{\prime}$ possesses maximum possible algebraic immunity 8 . This is the first demonstration of a Boolean function on an odd number of variables having nonlinearity greater than the bent concatenation bound and maximum possible algebraic immunity.

\section{Further improvement of nonlinearity}

Now we study the functions which are the outputs of Algorithm 1. We modify these functions to get 15 -variable balanced functions with nonlinearity better than 16264 . Let us first explain the theoretical idea behind this.

For this section, by $g$, we denote any function which is an output of Algorithm 1.

Theorem 2 Consider a function $g$. Let both of the maximum and second maximum absolute values in the Walsh spectrum of $g$ be negative in sign and let the values be $-v$ and $-v+\delta$, where $v, \delta>0$. Let $W_{g}(\omega)=-v$ for $\omega \in\left\{\omega^{(1)}, \ldots, \omega^{(t)}\right\}$. Consider the set $\left\{x^{(1)}, \ldots, x^{(s)}\right\}$ such that for any $x \in\left\{x^{(1)}, \ldots, x^{(s)}\right\}$, the values $\omega \cdot x$ are the same and $g(x)=1 \oplus \omega \cdot x$ for all $\omega \in\left\{\omega^{(1)}, \ldots, \omega^{(t)}\right\}$.

Consider $\left|\left\{x^{(1)}, \ldots, x^{(s)}\right\}\right| \geq \frac{\delta}{4}$ and let $Q$ be a $\frac{\delta}{4}$ size subset of $\left\{x^{(1)}, \ldots, x^{(s)}\right\}$. Construct

$$
\begin{aligned}
g^{\prime}(x) & =g(x) \text { for } x \in\{0,1\}^{n} \backslash Q, \\
& =1 \oplus g(x) \text { for } x \in Q .
\end{aligned}
$$

Then the maximum Walsh spectrum value of $g^{\prime}$ at the points $\omega \in\left\{\omega^{(1)}, \ldots, \omega^{(t)}\right\}$ will be the absolute value of $-v+\frac{\delta}{2}$ and the maximum absolute value of Walsh spectrum of $g^{\prime}$ will be $v-\frac{\delta}{2}$. If there exists an input point $\zeta$ with $W_{g}(\zeta)=0$ and $\sum_{x \in Q}(-1)^{g(x) \oplus \zeta \cdot x}=0$, then $W_{g^{\prime}}(\zeta)=0$.

Proof: For any $\omega \in\left\{\omega^{(1)}, \ldots, \omega^{(t)}\right\}$ and any $x \in Q$, we have

$$
(-1)^{g(x) \oplus \omega \cdot x}=(-1)^{1 \oplus \omega \cdot x \oplus \omega \cdot x}=-1 .
$$

Thus, $\sum_{x \in Q}(-1)^{g(x) \oplus \omega \cdot x}=-\frac{\delta}{4}$. Then,

$$
\begin{aligned}
W_{g^{\prime}}(\omega) & =\sum_{x \in\{0,1\}^{n} \backslash Q}(-1)^{g^{\prime}(x) \oplus \omega \cdot x}+\sum_{x \in Q}(-1)^{g^{\prime}(x) \oplus \omega \cdot x} \\
& =\sum_{x \in\{0,1\}^{n} \backslash Q}(-1)^{g(x) \oplus \omega \cdot x}-\left(\sum_{x \in Q}(-1)^{g(x) \oplus \omega \cdot x}\right) \\
& =W_{g}(\omega)-2\left(\sum_{x \in Q}(-1)^{g(x) \oplus \omega \cdot x}\right) \\
& =W_{g}(\omega)-2 \cdot\left(-\frac{\delta}{4}\right)=-v+\frac{\delta}{2} .
\end{aligned}
$$


Due to the toggling of $\frac{\delta}{4}$ output bits of $g$ to get $g^{\prime}$, other Walsh spectrum values $(-v+\delta$, the next maximum Walsh spectrum value in absolute terms) can be modified to $-v+\frac{\delta}{2}$ in $g^{\prime}$ (at most in absolute terms).

Again since, $W_{g}(\zeta)=0$ and $\sum_{x \in Q}(-1)^{g(x) \oplus \zeta \cdot x}=0$, then $\sum_{x \in\{0,1\}^{n} \backslash Q}(-1)^{g(x) \oplus \zeta \cdot x}=0$. Therefore

$$
\begin{aligned}
W_{g^{\prime}}(\zeta) & =\sum_{x \in\{0,1\}^{n} \backslash Q}(-1)^{g^{\prime}(x) \oplus \zeta \cdot x}+\sum_{x \in Q}(-1)^{g^{\prime}(x) \oplus \zeta \cdot x} \\
& =\sum_{x \in\{0,1\}^{n} \backslash Q}(-1)^{g(x) \oplus \zeta \cdot x}-\left(\sum_{x \in Q}(-1)^{g(x) \oplus \zeta \cdot x}\right) \\
& =0-0=0 .
\end{aligned}
$$

Note that the maximum absolute value in the Walsh spectrum of $g$ is 240 and the sign is negative. While modifying $g$ we will keep in mind the following points.

1. We attempt to toggle two output points of $g$ to get an increment of 2 in nonlinearity having one or more zeros in the Walsh spectrum. We refer to this modified function as $g^{\prime}$. The function $g^{\prime}$ is not an RSBF as this function will have two input orbits of size $>1$ where the outputs are not constant.

2. The points $\omega$ for which $W_{g}(\omega)=-240$ should provide $W_{g^{\prime}}(\omega)=-236$.

3. The points $\omega$ for which $W_{g}(\omega)=-236$ should provide $W_{g^{\prime}}(\omega)=-236$ or $W_{g^{\prime}}(\omega)=-232$. After toggling two points in the output of $g$, if we get $W_{g^{\prime}}(\omega)=-240$ for any such $\omega$, then the increment in nonlinearity will not be possible. Note that this issue can be avoided if there is no $\omega$ for which $W_{g}(\omega)=-236$. This is the reason we only consider the functions $g$ where there is no $\omega$ such that $W_{g}(\omega)=-236$. That is for each of these functions, the second maximum absolute value in the Walsh spectrum corresponds to -232 . We find that there are plenty of such functions among the 316 functions reported in the previous section.

4. For each of the functions $g$, the Walsh spectrum values are in the range [-240, 208]. The points $\omega$ for which $-232 \leq W_{g}(\omega) \leq 208$ will provide $-236 \leq W_{g^{\prime}}(\omega) \leq 212$ and they will not create any trouble if we want to have an increment in nonlinearity by 2 by toggling two output bits of $g$.

We select a function $g$ such that the second maximum absolute Walsh spectrum value of $g$ corresponds to -232. Referring to Appendix A will provide a handful of such functions. From the above argument it is clear that we need to concentrate on the $\omega$ 's for which $W_{g}(\omega)=-240$. Let us consider that there are $t$ such $\omega$ 's denoted by $\omega^{(1)}, \ldots, \omega^{(t)}$. We would like to get input points $x$ such that for all $\omega \in\left\{\omega^{(1)}, \ldots, \omega^{(t)}\right\}$ the values $\omega \cdot x$ are the same and also $g(x)=1 \oplus \omega \cdot x$. Say there are $s$ such input points $x^{(1)}, \ldots, x^{(s)}$. We choose two input points $x^{(i)}, x^{(j)}, 1 \leq i \neq j \leq s$ such that $(-1)^{g\left(x^{(i)}\right) \oplus \zeta \cdot x^{(i)}}+(-1)^{g\left(x^{(j)}\right) \oplus \zeta \cdot x^{(j)}}=0$ where $W_{g}(\zeta)=0$ and prepare $g^{\prime}$ as follows:

$$
\begin{aligned}
g^{\prime}(x) & =g(x) & & \text { when } x \in\{0,1\}^{n} \backslash\left\{x^{(i)}, x^{(j)}\right\} \\
& =1 \oplus g(x) & & \text { when } x \in\left\{x^{(i)}, x^{(j)}\right\} .
\end{aligned}
$$

Then following Theorem $2, g^{\prime}$ will have nonlinearity increased by 2 over that of $g$ as $W_{g^{\prime}}(\omega)=$ -236 for $\omega \in\left\{\omega^{(1)}, \ldots, \omega^{(t)}\right\}$ and for all other $\omega$ 's the maximum absolute value of $W_{g^{\prime}}(\omega)$ cannot exceed 236. Moreover the fact that $(-1)^{g\left(x^{(i)}\right) \oplus \zeta \cdot x^{(i)}}+(-1)^{g\left(x^{(j)}\right) \oplus \zeta \cdot x^{(j)}}=0$ will ensure that $W_{g^{\prime}}(\zeta)=0$. 
Construct the set $S$ of 15 -variable functions g with nonlinearity 16264 obtained by running Algorithm 1 such that the second maximum absolute value in the Walsh spectrum of each of them corresponds to -232 . We present the algorithm which takes a function $g \in S$ and returns a function with nonlinearity 16266 with some Walsh spectrum values equal to zero.

\section{Algorithm 2}

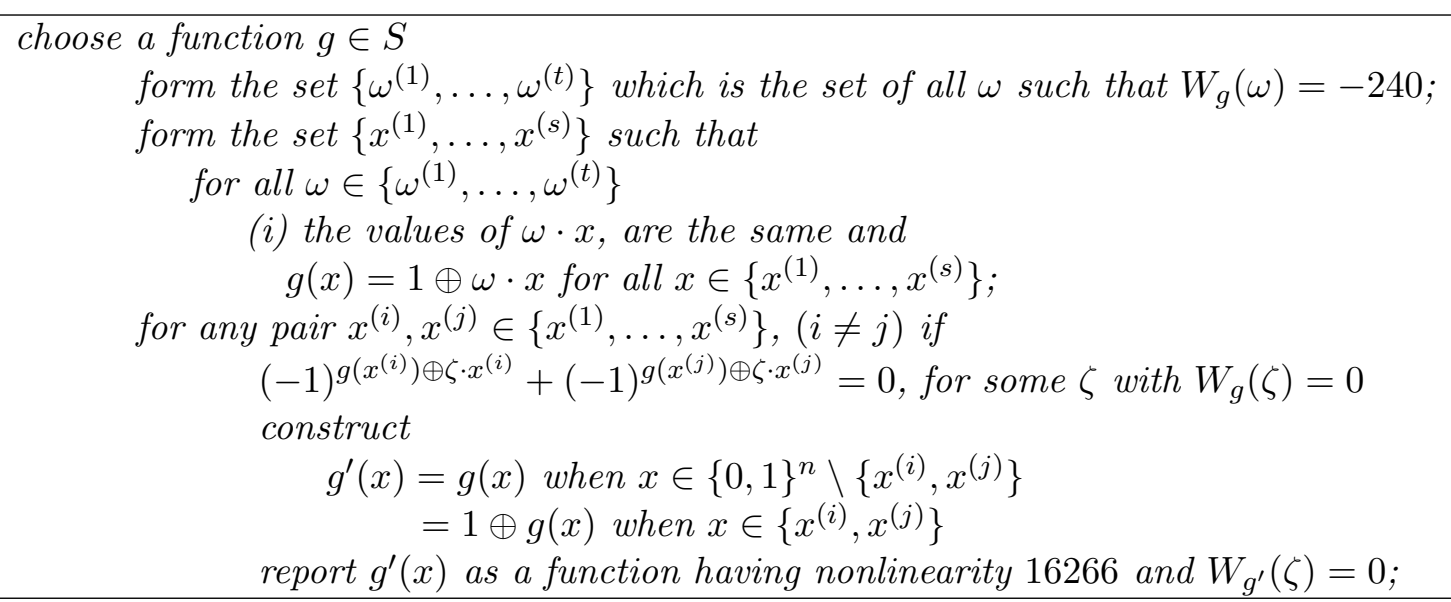

\section{Complexity of Algorithm 2}

Let $N=\left\{\omega^{(1)}, \ldots, \omega^{(t)}\right\}$. While forming the set $M=\left\{x^{(1)}, \ldots, x^{(s)}\right\}$, we require $N$ checks for each $x^{(i)} \in M$, i.e., in total $M \cdot N$ checks. Also to get the points $\zeta$ and $\omega$ such that $W_{g}(\zeta)=0$ and $W_{g}(\omega)=-240$, we require $O\left(n 2^{n}\right)$ time using the Fast Walsh Transform which is around $2^{19}$. Now for any pair $x^{(i)}, x^{(j)} \in\left\{x^{(1)}, \ldots, x^{(s)}\right\}$, the checking for $(-1)^{g\left(x^{(i)}\right) \oplus \zeta \cdot x^{(i)}}+$ $(-1)^{g\left(x^{(j)}\right) \oplus \zeta \cdot x^{(j)}}=0$, for $\zeta$ with $W_{g}(\zeta)=0$ requires constant time. Thus the total time complexity for Algorithm 2 is $\left(2^{19}+|M| \cdot|N|\right)<2^{19}+(2192)^{2}<2^{23}$. There are 232 functions $g$ in $S$. Therefore if we run Algorithm 2 for all the 232 functions $g$, the complexity will be around $2^{31}$.

Example 3 It is clear that the possibility of getting a larger set $\left\{x^{(1)}, \ldots, x^{(s)}\right\}$ increases when the size of the set $\left\{\omega^{(1)}, \ldots, \omega^{(t)}\right\}$ becomes smaller. In this manner we found functions $g$ such that $W_{g}(\omega)=-240$ is at only 30 points. We choose such a function $g$ with the following description. We consider the PW function having degree 8 and the function is transformed to an RSBF $f$ as described in Construction 1. The outputs of $f$ are toggled corresponding to the orbits represented by $(0,0,1,1,1,1,1,1,1,1,1,0,1,0,1)$ (of size 15) and $(0,1,0,1,1,0,1,0,1,1,0,1,0,1,1)$ (of size 5) to get $g$. Note that $n l(g)=16264$ and $\Delta_{g}=200$.

As per our description, we get $t=30$ points $\omega^{(1)}, \ldots, \omega^{(t)}$, for which the Walsh spectrum value of $g$ is -240 . Based on these we get $s=82$ many $x^{(1)}, \ldots, x^{(s)}$ and toggling the outputs of $g$ at any two of these 82 points increases the nonlinearity by 2 . As example we take the two input points $(0,0,0,0,0,1,1,0,0,1,1,1,0,0,1)$ and $(0,0,0,1,0,0,0,1,0,1,1,1,0,0,0)$ and toggle the outputs of $g$ at these two points to obtain $g^{\prime}$. The function $g^{\prime}$ has nonlinearity 16266 having 15 Walsh spectrum zeros, $\Delta_{g^{\prime}}=208$ and algebraic degree 14 . Next we construct the balanced functions $g^{\prime \prime}$, such that $g^{\prime \prime}(x)=g^{\prime}(x) \oplus \omega \cdot x$, where, $W_{g^{\prime}}(\omega)=0$. We choose such an $\omega=(0,0,0,1,1,1,0,1,1,0,0,1,0,1,0)$, and for this the function $g^{\prime \prime}(x)$ has maximum possible algebraic immunity equal to 8 .

Once we have $g^{\prime \prime}$, we can construct a balanced function on $n$-variables (odd $n>15$ ) as $b\left(x_{16}, \ldots, x_{n}\right) \oplus g^{\prime \prime}\left(x_{1}, \ldots, x_{15}\right)$ with nonlinearity $2^{n-1}-2^{\frac{n-1}{2}}+10 \times 2^{\frac{n-15}{2}}$, where $b$ is a bent function. 


\subsection{Currently best known results}

Here we present the results that could be found after the publication of the conference version of this paper [33]. In [16], the PW functions having nonlinearity 16268 [25] are studied. Directed search has been exploited in [16] to toggle the outputs corresponding to 20 orbits (13 of size 15,5 of size 5,2 of size 1) to get a balanced function without any reduction in nonlinearity. Note that, in our technique we motivate the exhaustive search in the neighbourhood of the PW functions. Exhaustive search considering 20 orbits is computationally infeasible, but the kind of directed search [16] motivated by our technique may provide more improved results.

The next challenge is to get 15 -variable balanced functions with nonlinearity more than 16268 (by searching the neighbourhood of PW functions, but not by some heuristics search). So far we have considered the neighbourhood of a PW function having nonlinearity 16276 by modifying one orbit of size 5 and another of size 15 . The next motivation is to extend the neighbourhood further and we studied the neighbourhood considering three orbits of size 15. Thus there are $\left(\begin{array}{c}2182 \\ 3\end{array}\right)<2^{31}$ many options. We studied this space and found unbalanced functions with nonlinearity 16271, having 2 as the minimum absolute value in the Walsh spectrum. Then we considered one of the two size 1 orbits to make it balanced and in the process the nonlinearity is increased further to $16272^{1}$. As example, an RSBF $g_{1}$ is obtained by toggling the outputs of $f$ (degree 8 PW function having nonlinearity 16276) at the orbits $315,2275,8183$ (of size 15) and 0 (of size 1). Consider the decimal values as 15-bit binary patterns. The function $g_{1}$ has 7 zeros in its Walsh spectrum with the parameters $\Delta_{g_{1}}=248$ and algebraic degree 11. Consider the input $\omega=4681$ as one of the points such that $W_{g_{1}}(\omega)=0$. Construct the function $g_{2}(x)=g_{1}(x) \oplus \omega \cdot x$. Then $g_{2}$ is balanced with $n l\left(g_{2}\right)=16272$ and $\Delta_{g_{2}}=248$. We have checked that the algebraic immunity of $g_{3}$ is equal to 8 . This demonstrates a Boolean function on an odd number of variables having nonlinearity greater than the bent concatenation bound and maximum possible algebraic immunity. Refer to Appendix $\mathrm{C}$ for the truth table representation of this paper.

\section{Strategy to get 1-resilient functions}

Any 15-variable RSBF $g$ with nonlinearity 16264 from the list of Appendix A (i.e., output of Algorithm 1) has 15 many zeros and all of these 15 input points with Walsh spectrum zeros belong to one orbit of size 15 . Now one may note that for an $n$-variable 1-resilient function, the number of Walsh spectrum zeros is at least $n+1$. Thus the functions from Appendix A cannot be affinely transformed to 1-resilient functions. To get more Walsh spectrum zeros, we need to modify the functions further. We consider the additional points where the Walsh spectrum values are close to zero. We observe that the value in the Walsh spectrum closest to zero is 16 which occurs for some functions of Appendix A, also for each of these functions the Walsh spectrum value 16 occurs at one or more orbits of size 15 only. We construct the set $S^{\prime}$ which constitutes the functions $g$ such that the second minimum Walsh spectrum value is 16 . We would like to modify any function from $S^{\prime}$ such that

1. the existing orbit with Walsh spectrum value zero stays at zero and

2. one or more of the existing orbits with Walsh spectrum value 16 drop to zero.

This strategy will indeed increase the Walsh spectrum zeros in the modified function. The only issue that has to be noted is the drop in nonlinearity after this modification. As the nonlinearity

\footnotetext{
${ }^{1}$ One of the reviewers of a recent version of this paper has also pointed out this neighbourhood by identifying a nonlinearity 16268 balanced function.
} 
of 1-resilient functions must be divisible by four [31] and we are interested in nonlinearities greater than the bent concatenation bound 16256, the nonlinearities of the modified functions should be 16260 or 16264 (or even more, but we actually did not get more than that in the experimentation we did).

Theorem 3 Consider a function $g \in S^{\prime}$ such that $W_{g}\left(\Lambda_{n, p}\right)=0$ and $W_{g}\left(\Lambda_{n, j}\right)=16$. Let

1. $(-1)^{f\left(\Lambda_{n, q}\right)}{ }_{n} \mathcal{A}_{q, j}+(-1)^{f\left(\Lambda_{n, r}\right)}{ }_{n} \mathcal{A}_{r, j}=8$, and

2. $(-1)^{f\left(\Lambda_{n, q}\right)}{ }_{n} \mathcal{A}_{q, p}+(-1)^{f\left(\Lambda_{n, r}\right)}{ }_{n} \mathcal{A}_{r, p}=0$,

where $\Lambda_{n, q}, \Lambda_{n, r}$ are two orbit representative elements. Construct

$$
\begin{aligned}
h(x) & =g(x) \text { for } x \in\{0,1\}^{n} \backslash G_{n}\left(\Lambda_{n, q}\right) \cup G_{n}\left(\Lambda_{n, r}\right), \\
& =1 \oplus g(x) \text { for } x \in G_{n}\left(\Lambda_{n, q}\right) \cup G_{n}\left(\Lambda_{n, r}\right),
\end{aligned}
$$

then $W_{h}\left(\Lambda_{n, j}\right)=W_{h}\left(\Lambda_{n, p}\right)=0$.

Proof: Since, $W_{g}\left(\Lambda_{n, j}\right)=16$ and $(-1)^{g\left(\Lambda_{n, q}\right)}{ }_{n} \mathcal{A}_{q, j}+(-1)^{g\left(\Lambda_{n, r}\right)}{ }_{n} \mathcal{A}_{r, j}=8$, therefore, $\sum_{i \notin\{q, r\}}(-1)^{g\left(\Lambda_{n, i}\right)}{ }_{n} \mathcal{A}_{i, j}=8$. Now,

$$
\begin{aligned}
W_{h}\left(\Lambda_{n, j}\right) & =\sum_{i \notin\{q, r\}}(-1)^{h\left(\Lambda_{n, i}\right)}{ }_{n} \mathcal{A}_{i, j}+(-1)^{h\left(\Lambda_{n, q}\right)}{ }_{n} \mathcal{A}_{q, j}+(-1)^{h\left(\Lambda_{n, r}\right)}{ }_{n} \mathcal{A}_{r, j} \\
& =\sum_{i \notin\{q, r\}}(-1)^{g\left(\Lambda_{n, i}\right)}{ }_{n} \mathcal{A}_{i, j}-(-1)^{g\left(\Lambda_{n, q}\right)}{ }_{n} \mathcal{A}_{q, j}-(-1)^{g\left(\Lambda_{n, r}\right)}{ }_{n} \mathcal{A}_{r, j} \\
& =8-8=0 .
\end{aligned}
$$

Again since, $W_{g}\left(\Lambda_{n, p}\right)=0$ and $(-1)^{f\left(\Lambda_{n, q}\right)}{ }_{n} \mathcal{A}_{q, p}+(-1)^{f\left(\Lambda_{n, r}\right)}{ }_{n} \mathcal{A}_{r, p}=0$, the proof that $W_{h}\left(\Lambda_{n, p}\right)=0$ follows easily by the similar argument as given above.

We consider a function $g \in S^{\prime}$. Then the orbit $G_{n}\left(\Lambda_{n, p}\right)$ such that $W_{g}\left(\Lambda_{n, p}\right)=0$ is of size 15 and also the orbits $G_{n}\left(\Lambda_{n, j}\right)$ such that $W_{g}\left(\Lambda_{n, j}\right)=16$ are of size 15 . Now we form the sets $\left\{q_{1}, \ldots, q_{t}\right\}$ and $\left\{r_{1}, \ldots, r_{l}\right\}$ such that for each $q \in\left\{q_{1}, \ldots, q_{t}\right\}$ and $r \in\left\{r_{1}, \ldots, r_{l}\right\}$, we have, $\left|{ }_{n} \mathcal{A}_{q, j}\right|=5$ and $\left|{ }_{n} \mathcal{A}_{r, j}\right|=3$. Then we consider those pairs for which

1. $(-1)^{f\left(\Lambda_{n, q}\right)}{ }_{n} \mathcal{A}_{q, j}+(-1)^{f\left(\Lambda_{n, r}\right)}{ }_{n} \mathcal{A}_{r, j}=8$, and

2. $(-1)^{f\left(\Lambda_{n, q}\right)}{ }_{n} \mathcal{A}_{q, p}+(-1)^{f\left(\Lambda_{n, r}\right)}{ }_{n} \mathcal{A}_{r, p}=0$.

Then by Theorem 3 , we have $W_{h}\left(\Lambda_{n, j}\right)=W_{h}\left(\Lambda_{n, p}\right)=0$. Thus the modified function $h$ will have at least 30 zeros in its Walsh spectrum. Due to this modification, nonlinearity may fall. However we intend to keep functions $h$ which have nonlinearity more than the bent concatenation bound 16256 and divisible by 4 (as a 1-resilient function must have its nonlinearity divisible by 4). Based on this discussion we present the following algorithm.

\section{Algorithm 3}


$n=15$

choose a function $g \in S^{\prime}$;

find an orbit representative $\Lambda_{n, j}$ such that $W_{g}\left(\Lambda_{n, j}\right)=16$;

find the orbit representative $\Lambda_{n, p}$ such that $W_{g}\left(\Lambda_{n, p}\right)=0$;

form the set $\left\{q_{1}, \ldots, q_{t}\right\}$ and $\left\{r_{1}, \ldots, r_{l}\right\}$ such that

$\left|{ }_{n} \mathcal{A}_{q, j}\right|=5$ and $\left|{ }_{n} \mathcal{A}_{r, j}\right|=3$ for all $q \in\left\{q_{1}, \ldots, q_{t}\right\}$ and $r \in\left\{r_{1}, \ldots, r_{l}\right\}$

for each $q \in\left\{q_{1}, \ldots, q_{t}\right\}$ and for each $r \in\left\{r_{1}, \ldots, r_{l}\right\}$ if

1. $(-1)^{g\left(\Lambda_{n, q}\right)}{ }_{n} \mathcal{A}_{q, j}+(-1)^{g\left(\Lambda_{n, r}\right)}{ }_{n} \mathcal{A}_{r, j}=8$

2. $(-1)^{g\left(\Lambda_{n, q}\right)}{ }_{n} \mathcal{A}_{q, p}+(-1)^{g\left(\Lambda_{n, r}\right)}{ }_{n} \mathcal{A}_{r, p}=0$

Construct

$h(x)=g(x)$ for $x \in\{0,1\}^{n} \backslash G_{n}\left(\Lambda_{n, q}\right) \cup G_{n}\left(\Lambda_{n, r}\right)$,

$=1 \oplus g(x)$ for $x \in G_{n}\left(\Lambda_{n, q}\right) \cup G_{n}\left(\Lambda_{n, r}\right)$;

if $n l(h) \geq 16260$ and 4 divides $n l(g)$

store $h$ in file $F$;

file $F$ provides 15 -variable functions with nonlinearity $\geq 16260$

and having Walsh spectrum zeros in at least 30 points for each of the functions;

\section{Complexity of Algorithm 3}

The computational effort of this algorithm depends on the number of orbits $G_{n}\left(\Lambda_{n, q}\right)$ and $G_{n}\left(\Lambda_{n, r}\right)$ such that $\left|{ }_{n} \mathcal{A}_{q, j}\right|=5$ and $\left|{ }_{n} \mathcal{A}_{r, j}\right|=3$, i.e., we have to check $t \times l$ many options which can attain the maximum value $\left(\frac{2192}{2}\right)^{2}$. Also within the loop, determination of the nonlinearity of the modified function $h$ requires $O\left(n 2^{n}\right)$, i.e., around $2^{19}$ time by using Fast Walsh Transform. Thus total complexity for Algorithm 2 is $t \times l \times 2^{19}<\left(\frac{2192}{2}\right)^{2} \times 2^{19}<2^{40}$. There are 292 functions $g \in S^{\prime}$. Therefore if one wishes to run Algorithm 3 for all these functions, the time complexity will be less than $2^{49}$.

Given an $n$-variable Boolean function $\phi$, let us define

$$
S_{\phi}=\left\{\omega \in\{0,1\}^{n} \mid W_{\phi}(\omega)=0\right\} .
$$

If there exist $n$ linearly independent vectors in $S_{\phi}$, then one can construct a nonsingular $n \times n$ matrix $B_{\phi}$ whose rows are linearly independent vectors from $S_{\phi}$. Let, $C_{\phi}=B_{\phi}^{-1}$. Now one can define $\phi^{\prime}(x)=\phi\left(C_{\phi} x\right)$. Both $\phi^{\prime}$ and $\phi$ have the same weight, nonlinearity and algebraic degree. Moreover, $W_{\phi^{\prime}}(\omega)=0$ for $w t(\omega)=1$. This ensures that $\phi^{\prime}$ is correlation immune of order 1 . Further if $\phi$ is balanced then $\phi^{\prime}$ is 1-resilient. This technique has been used in [18,24].

We run Algorithm 3 for few functions $g \in S^{\prime}$. In the following example we describe it.

Example 4 Let $n=15$. We consider the RSBF $f$ obtained from the 8-degree PW function using Construction 1. We run Algorithm 3 for a small subset of $S^{\prime}$. We take functions $g \in S^{\prime}$ obtained from $f$ such that the value 16 occurs exactly at 15 points in the Walsh spectrum. For these functions we find 32066 functions with nonlinearity either 16260 or 16264 and having at least 30 Walsh zeros. For example, we take a function $g \in S^{\prime}$ which is obtained by toggling the outputs of $f$ corresponding to the orbits of size 15 and 5 having representative elements respectively $(0,0,0,0,0,1,1,1,0,1,0,0,1,1,1)$ and $(0,1,0,1,1,0,1,0,1,1,0,1,0,1,1)$. We have $n l(g)=16264$ and $W_{g}$ contains 15 many zeros. We apply Algorithm 3 over the function $g$ to get $h$ such that $n l(h)=16264$ and $W_{h}$ contains 135 many zeros. The function $h$ is obtained by toggling the outputs of $g$ corresponding to the orbits represented by the tuples $(0,0,1,1,0,1,0,1,1,1,0,1,1,1,1)$ and $(0,0,0,0,1,1,1,1,0,1,1,0,0,1,1)$ each of size 15 . We note that $W_{h}(\omega)=0$ for $\omega=(0,0,0,0,0,1,0,0,0,0,1,1,0,1,1)$. Thus the function $\phi=h \oplus \omega \cdot x$ will be balanced. Then as described above, we find 15 linearly independent vectors from $S_{\phi}$ and hence a 1-resilient function $\phi^{\prime}$ having nonlinearity 16264 is found. We note that for $\phi^{\prime}, \Delta_{\phi^{\prime}}=$ 
232 with algebraic degree 12 and algebraic immunity 7. See Appendix B for the truth table of this function. This shows for the first time the existence of a 1-resilient function exceeding the bent concatenation bound in nonlinearity with maximum absolute autocorrelation value less than $2^{\frac{15+1}{2}}$.

In [15], existence of 1-resilient functions having the maximum absolute value in the autocorrelation spectra $<2^{\frac{n+1}{2}}$ has been demonstrated for $n=9,11$. However, the nonlinearity in those cases did not exceed the bent concatenation bound.

In $[30,32]$, a method to construct resilient functions on odd numbers of variables, having nonlinearity greater than the bent concatenation bound, has been proposed. The construction used the PW functions as a part of it. In the process, a 41-variable 1-resilient function $\psi_{1}$ has been designed with $n l\left(\psi_{1}\right)>2^{40}-2^{20}+51 \times 2^{10}$. Thus so far, the resilient functions, having nonlinearity greater than the bent concatenation bound, had been known for 41 or more variables. Example 4 above shows the existence of a 15-variable function with nonlinearity that exceeds the bent concatenation bound. Again for odd $n>15$, the function $b\left(x_{16}, \ldots, x_{n}\right) \oplus$ $\phi^{\prime}\left(x_{1}, \ldots, x_{15}\right)$, where $b\left(x_{16}, \ldots, x_{n}\right)$ is a bent function, will be 1-resilient with nonlinearity $2^{n-1}-2^{\frac{n-1}{2}}+8 \times 2^{\frac{n-15}{2}}$. This shows that 1-resilient functions are available for 15 or more variables. Thus the gap between 15 to 39 variables is now settled. Further we show that using the function $\phi^{\prime}$ we can construct a 41-variable 1-resilient function with nonlinearity that exceeds the lower bound of $n l\left(\psi_{1}\right)$. Let $\psi_{2}=b\left(x_{16}, \ldots, x_{41}\right) \oplus \phi^{\prime}\left(x_{1}, \ldots, x_{15}\right)$, where $b\left(x_{16}, \ldots, x_{41}\right)$ is a bent function, then $n l\left(\psi_{2}\right)=2^{40}-2^{20}+8 \times 2^{\frac{41-15}{2}}=2^{40}-2^{20}+64 \times 2^{10}$ which is greater than $2^{40}-2^{20}+51 \times 2^{10}$, the lower bound of $n l\left(\psi_{1}\right)$.

\section{Conclusion}

In this paper we successfully modify the two 15-variable PW functions [25] to construct balanced functions $f$ with currently best known nonlinearity (16272) and autocorrelation parameters. Some of these functions provide the maximum algebraic immunity 8 . These results improve the parameters presented in $[16,19,30]$. Further we could also construct 1-resilient functions on 15-variables having nonlinearity 16264 that were not known earlier. The 1-resilient functions on odd number of variables having nonlinearity greater than the bent concatenation bound were earlier known for 41 or more variables [30,32]. Apart from the improvements in the parameter values, the theoretical contribution of this paper is to modify any of the PW functions keeping their idempotent structure unchanged and inducing Walsh spectrum zeros in the modified function. Given balancedness, 1-resiliency, maximum possible algebraic immunity, very good nonlinearity and nice autocorrelation properties, we recommend use of these functions in cipher design.

\section{References}

[1] Berlekamp, E.R., Welch, L.R.: Weight distributions of the cosets of the (32,6) Reed-Muller code. IEEE Transactions on Information Theory 18(1), 203-207 (1972)

[2] Carlet, C., Dalai, D.K., Gupta, K.C., Maitra, S.: Algebraic immunity for cryptographically significant Boolean functions: Analysis and construction. IEEE Transactions on Information Theory 52(7), 3105-3121 (2006) 
[3] Courtois, N., Meier, W.: Algebraic attacks on stream ciphers with linear feedback. In: E. Biham (ed.) EUROCRYPT, Lecture Notes in Computer Science, vol. 2656, pp. 345-359. Springer (2003)

[4] Cusick, T.W., Stănică, P.: Fast evaluation, weights and nonlinearity of rotation-symmetric functions. Discrete Mathematics 258(1-3), 289-301 (2002)

[5] Dalai, D.K., Gupta, K.C., Maitra, S.: Results on rotation symmetric bent functions. In: Second International Workshop on Boolean Functions: Cryptography and Applications, BFCA'06, pp. 107-124. Publications of Universities of Rouen and Havre (2006)

[6] Dalai, D.K., Maitra, S., Sarkar, S.: Results on rotation symmetric bent functions. In: Second International Workshop on Boolean Functions: Cryptography and Applications, BFCA'06, pp. 137-156. Publicaions of Universities of Rouen and Havre (2006)

[7] Dillon, J.F.: Elementary hadamard difference sets. Ph.D. thesis, University of Maryland (1974)

[8] Dobbertin, H.: Construction of bent functions and balanced Boolean functions with high nonlinearity. In: B. Preneel (ed.) Fast Software Encryption, Lecture Notes in Computer Science, vol. 1008, pp. 61-74. Springer (1994)

[9] Filiol, E., Fontaine, C.: Highly nonlinear balanced Boolean functions with a good correlation-immunity. In: K. Nyberg (ed.) EUROCRYPT, Lecture Notes in Computer Science, vol. 1403, pp. 475-488. Springer (1998)

[10] Fontaine, C.: The nonlinearity of a class of Boolean functions with short representation. In: J. Přribyl (ed.) PRAGOCRYPT'96, pp. 129-144. CTU Publishing House (1996)

[11] Fontaine, C.: On some cosets of the first-order Reed-Muller code with high minimum weight. IEEE Transactions on Information Theory 45(4), 1237-1243 (1999)

[12] Hell, M., Maximov, A., Maitra, S.: On efficient implementation of search strategy for rotation symmetric Boolean functions. In: Proceedings of Ninth International Workshop on Algebraic and Combinatoral Coding Theory, ACCT 2004, pp. 19-25 (2004)

[13] Kavut, S., Maitra, S., Sarkar, S., Yücel, M.D.: Enumeration of 9-variable rotation symmetric Boolean functions having nonlinearity $>240$. In: R. Barua, T. Lange (eds.) INDOCRYPT, Lecture Notes in Computer Science, vol. 4329, pp. 266-279. Springer (2006)

[14] Kavut, S., Maitra, S., Yücel, M.D.: Autocorrelation spectra of balanced Boolean functions on odd number input variables with maximum absolute value $<2^{\frac{n+1}{2}}$. In: Second International Workshop on Boolean Functions: Cryptography and Applications, BFCA 06, pp. 73-86. Publications of Universities of Rouen and Havre (2006)

[15] Kavut, S., Maitra, S., Yücel, M.D.: Search for Boolean functions with excellent profiles in the rotation symmetric class. IEEE Transactions on Information Theory 53(5), 1743-1751 (2007)

[16] Kavut, S., Yücel, M. D.: Balanced Boolean Functions with Nonlinearity $>2^{n-1}-2^{\frac{n-1}{2}}$. Cryptology ePrint Archive, http://eprint.iacr.org/, Report 2007/321, 15 August, 2007. 
[17] Langevin, P., Zanotti, J. -P.: Nonlinearity of some invariant Boolean functions. Designs, Codes and Cryptography 36(2), 131-146 (2005)

[18] Maitra, S., Sarkar, P.: Cryptographically significant Boolean functions with five valued walsh spectra. Theoretical Computer Sciience 276(1-2), 133-146 (2002)

[19] Maitra, S., Sarkar, P.: Modifications of patterson-wiedemann functions for cryptographic applications. IEEE Transactions on Information Theory 48(1), 278-284 (2002)

[20] Maximov, A.: Classes of plateaued rotation symmetric Boolean functions under transformation of walsh spectra. In: International Workshop on Coding and Cryptography, WCC 2005, pp. 325-334 (2005)

[21] Maximov, A., Hell, M., Maitra, S.: Plateaued rotation symmetric Boolean functions on odd number of variables. In: First International Workshop on Boolean Functions: Cryptography and Applications, BFCA 05, pp. 83-104. Publications of Universities of Rouen and Havre (2005)

[22] Meier, W., Pasalic, E., Carlet, C.: Algebraic attacks and decomposition of Boolean functions. In: C. Cachin, J. Camenisch (eds.) EUROCRYPT, Lecture Notes in Computer Science, vol. 3027, pp. 474-491. Springer (2004)

[23] Mykkeltveit, J.J.: The covering radius of the $(128,8)$ Reed-Muller code is 56. IEEE Transactions on Information Theory 26(3), 359-362 (1980)

[24] Pasalic, E., Johansson, T.: Further results on the relation between nonlinearity and resiliency for Boolean functions. In: M. Walker (ed.) IMA International Conference, Lecture Notes in Computer Science, vol. 1746, pp. 35-44. Springer (1999)

[25] Patterson, N.J., Wiedemann, D.H.: The covering radius of the $\left(2^{15}, 16\right)$ Reed-Muller code is at least 16276. IEEE Transactions on Information Theory 29(3), 354-356 (1983). See also the correction in [26]

[26] Patterson, N.J., Wiedemann, D.H.: Correction to 'the covering radius of the $\left(2^{15}, 16\right)$ Reed-Muller code is at least 16276' (may 83 354-356). IEEE Transactions on Information Theory 36(2), 443 (1990)

[27] Pieprzyk, J., Qu, C.X.: Rotation-symmetric functions and fast hashing. Jornal of Universal Computer Science 5(1), 20-31 (1999)

[28] Preneel, B., Leekwijck, W.V., Linden, L.V., Govaerts, R., Vandewalle, J.: Propagation characteristics of Boolean functions. In: Advances in Cryptology-EUROCRYPT, Lecture Notes in Computer Science, vol. 473, pp. 161-173 (1990)

[29] Rothaus, O.S.: On "bent" functions. Journal of Combinatorial Theory, Series A 20(3), 300-305 (1976)

[30] Sarkar, P., Maitra, S.: Construction of nonlinear Boolean functions with important cryptographic properties. In: Advances in Cryptology-EUROCRYPT, Lecture Notes in Computer Science, vol. 1807, pp. 485-506 (2000)

[31] Sarkar, P., Maitra, S.: Nonlinearity bounds and constructions of resilient Boolean functions. In: M. Bellare (ed.) CRYPTO, Lecture Notes in Computer Science, vol. 1880, pp. 515-532. Springer (2000) 
[32] Sarkar, P., Maitra, S.: Construction of nonlinear resilient Boolean functions using "small" affine functions. IEEE Transactions on Information Theory 50(9), 2185-2193 (2004)

[33] Sarkar, S., Maitra, S.: Idempotents in the Neighbourhood of Patterson-Wiedemann Functions having Walsh Spectra Zeros. In WCC 2007, International Workshop on Coding and Cryptography, Pages 351-360, April 16-20, 2007, Versailles (France)

[34] Seberry, J., Zhang, X., Zheng, Y.: Nonlinearly balanced Boolean functions and their propagation characteristics (extended abstract). In: D.R. Stinson (ed.) CRYPTO, Lecture Notes in Computer Science, vol. 773, pp. 49-60. Springer (1993)

[35] Stănică, P., Maitra, S.: Rotation symmetric Boolean functions - count and cryptographic properties. In: R. C. Bose Centenary Symposium on Discrete Mathematics and Applications, Electronic Notes in Discrete Mathematics, vol. 15. Elsevier (2002)

[36] Stănică, P., Maitra, S., Clark, J.A.: Results on rotation symmetric bent and correlation immune Boolean functions. In: B.K. Roy, W. Meier (eds.) FSE, Lecture Notes in Computer Science, vol. 3017, pp. 161-177. Springer (2004)

[37] Xiao, G.Z., Massey, J.L.: A spectral characterization of correlation-immune combining functions. IEEE Transactions on Information Theory 34(3), 569-571 (1988)

[38] Zhang, K., Zheng, Y.: Gac - the criterion for global avalance characteristics of cryptographic functions. Jornal of Universal Computer Science 1(5), 320-337 (1995) 


\section{Appendix A}

In Table 3, 253 pairs in the upper part and 63 pairs in the lower part correspond to 15 -variable functions with nonlinearity 16264 obtained respectively from the 9-degree and 8-degree PW functions. In each pair the first integer indicates the decimal value of the representative of the orbit of size 15 and the second one indicates the decimal value of the representative of the orbit of size 5 . The decimal value corresponding to an $n$-bit binary vector $\left(x_{n}, x_{n-1}, \ldots, x_{1}\right) \in$ $\{0,1\}^{n}$ is determined by taking $x_{n}$ as the most significant bit. Pairs marked $\left(^{*}\right)$ indicate the functions with the second maximum absolute Walsh spectrum value corresponding to -232 . Pairs marked $(+)$ indicate functions having 16 in their Walsh spectra.

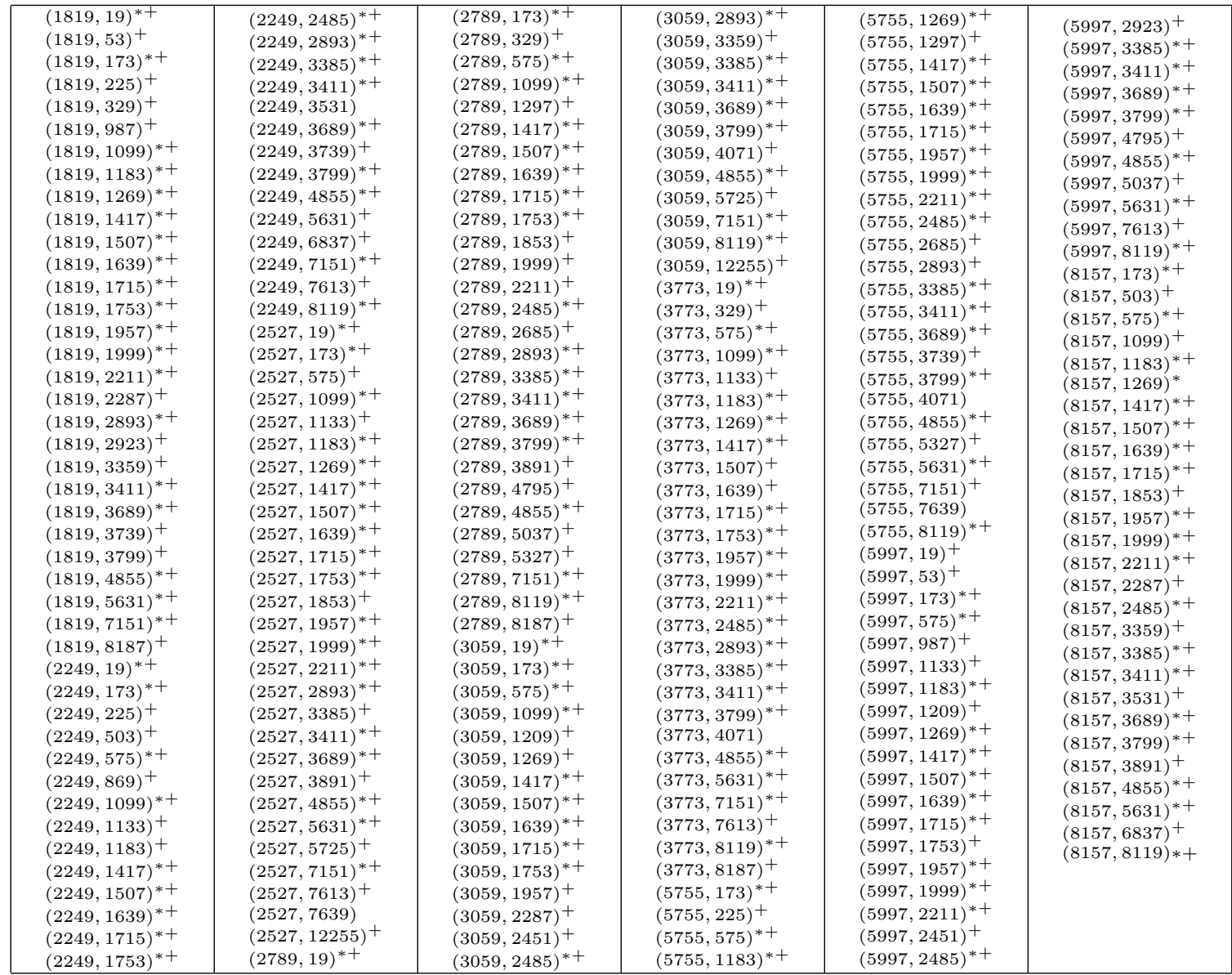

\begin{tabular}{|c|c|c|c|c|}
\hline $\begin{array}{l}(181,1057)^{*} \\
(181,7399)^{*+} \\
(181,11627)^{*+} \\
(595,1057)^{*} \\
(595,7399)^{*+} \\
(595,11627)^{*} \\
(767,1057)^{*} \\
(767,7399)^{*+} \\
(767,11627)^{*+} \\
(861,1057)^{*+} \\
(861,7399)^{*} \\
(861,11627)^{*+} \\
(935,1057)^{*+}\end{array}$ & $\begin{array}{l}(935,7399)^{*+} \\
(935,11627)^{*+} \\
(1165,1057)^{*} \\
(1165,7399)^{*+} \\
(1165,11627)^{*+} \\
(1401,1057)^{*} \\
(1401,7399)^{*+} \\
(1401,11627)^{*} \\
(1493,1057)^{*+} \\
(1493,7399)^{*+} \\
(1493,11627)^{*+} \\
(1643,1057)^{*+} \\
(1643,7399)^{*}\end{array}$ & $\begin{array}{l}(1643,11627)^{*+} \\
(1843,1057)^{*+} \\
(1843,7399)^{*+} \\
(1843,11627)^{*+} \\
(1993,1057)^{*+} \\
(1993,7399)^{*+} \\
(1993,11627)^{*+} \\
(2861,1057)^{*} \\
(2861,7399)^{*+} \\
(2861,11627)^{*+} \\
(2939,1057)^{*} \\
(2939,7399)^{*+} \\
(2939,11627)^{*+}\end{array}$ & $\begin{array}{l}(3493,1057)^{*+} \\
(3493,7399)^{*+} \\
(3493,11627)^{*+} \\
(4097,1057)^{*+} \\
(4097,7399)^{*} \\
(4097,11627)^{*+} \\
(4837,1057)^{*+} \\
(4837,7399)^{*+} \\
(4837,11627)^{*+} \\
(5053,1057)^{*} \\
(5053,7399)^{*+} \\
(5053,11627)^{*+} \\
(5099,1057)^{*}\end{array}$ & $\begin{array}{l}(5099,7399)^{*+} \\
(5099,11627)^{*+} \\
(6967,1057)^{*} \\
(6967,7399)^{*+} \\
(6967,11627)^{*+} \\
(8181,1057)^{*} \\
(8181,7399)^{*+} \\
(8181,11627)^{*+} \\
(10939,1057)^{*} \\
(10939,7399)^{*+} \\
(10939,11627)^{*}\end{array}$ \\
\hline
\end{tabular}

Table 3: 15-variable functions with nonlinearity 16264 obtained from 9 and 8-degree PW functions 


\section{Appendix B}

Truth table of the 15-variable 1-resilient function with nonlinearity 16264, degree 12, maximum autocorrelation value 232 and algebraic immunity 7 as given in Example 4 is as follows:

49D1CCFBCED8C4BD485BAE6B5D0E130BEAEAEDB7E9016569171EE5C63C43B9D60AC22A8B5BD2E025E5FFDB6C2AA57201413D498087AE07293F62DFA77BDDAB69 FE02CE542FE83C702D6F13B811BBF1463057171C79FE868BBE3549660711D14CB9DFA6A727E860AA0752AD37CCF7F5F887232FD8417BA58C7E3DCB1B8BCD299 C2E4EB2E44CA090752EC400E75936CF9D625BAOAD413EBFE41B34873DD1BCD44A7F64EA5BA7D5A2789B11A774F141062D60B9710B139781D9AF5D99E47E4DB19 6A9D72DEFDDA06230DCCA45A1CB8FA9B2788CDD610ED0E6E29129B72BOEA2ADCB195AB7551799726DOF06E869A5850CE36756E9A82E6FE56E944BEC62B802B74 C36FA87BA2674837FB09B73DDF6C74DD3D65E3EDA4E65F95524F3E4B8AF3B2EDE54385FDB1EB5E98D8B87EFDD1C2C4EDC40F6109C79C6026471994B6D6BD5EDB C87FD6CA40952939B7B2E2DB5BBD1B8E9414126E92582400E9963B152B8B554889F413607D75CFDFFD9CE2D53CF86B95C4E2E5B235F0E8F4266EBE1D156A399 3307F97EA963B49BOFE40067450B10EBB44D97C2E0581500D04AD9C7EDE492BB4925FA9042802EB355251BF3B9DD1366BE6F9D925216924EF67E343EFC663206 70A3030536EC545D6C16308F70ACC8D7261A948EE1CD594F2C8188263C19F3DE2D18C1FA4D051C7DF91C63D512F31E33136994C02EE307A43B09D02EF3BCED6E 496434E025159CD0C78F8444A495FA702F3FD43C5992BF7058287C7D4CADB45E862630E173E1B3B3922B3F13DC6879B60F1EE672AF85A7267E87A90CBC098A84 113362C0110A307D88758E2FAE116379FB3D505B4EBA461CCCE37C5DAE54C8759AFBC676920BE19CFB89BB2443E54CA3E45F13CAD45501E2061F7AE5BD766A4F 3A21153F04E11F6F2D231C9CA95C741DA1BE00F8258CB711AFFAF8D1128473BD332B08F158AD69830BA7F402190CA7490C93BCEB352013F5844D03EEDF9D2450 BEB81490DFA69C5F9688D25A624812D2BAD31CF4F919DC742358789A19B5D8F211BB3F52BD866B8907008AFEFB8439F47FDD49087EA672E3F1D0D9E630B85F22 E9A0CA2E201D6A74C8FC949A8851F8EC15F1E3AF9340859ABB5AD8989B06EA30DA2D2AC7A0CA6E2850327D72E8C7770AD5EA7884E12A49BE0F5A83E62EABC8E5 1DC0429F00CB57798DCD3D33D091D43EFA3BFF17EF63F4F118836A45D7DCED2267EA93AA7069BAB2A4A2AE7089581974E6B387116B3184ED53D361D7FE4D252B 07E440 1 1001 758D61 D34D21E91DF685C9151538B9CC43DCF1052A8E9F43172747EAOE2A414C589CAAD2EAEF9823D79841802414AC61C8882C02C328BF90767467F62DA785E7FBE96 DDB07B23A 9 (1) E698D48BC549 1BE9082B35BD5897059C1AF9150F2D6B4740770070346F38919FEE353212F7AB3E2B5FDFD0FB25B2FFD4FA0B7A565CFB5C00108D745A8D5162D1DAEDF1A37FA8 428862454CB17D652957560F7D9213F326B5D9929F71D93BDDFB7D21DA580045E417D249D6215B1C2F1F391DBCDF124E79CD6F3CB9925F7E74092E45F0233E93 9D04AF08A0CB9816564758C16EBFF7BC5BABC26C7816C8775ECEC598E7166C2111C8C6FD3240B765F16CA1AD2923F4798BEB5CB37DA3852BB3FCE30C80C9C47 13F5ACA22EB9AF335A1285E500634B99DC1B8A188B64AB19E30019B452F41A8C3E8DAD8B2A83BB15E9F3DC540F25CA79919BDE418B5058F6772599B4352F051 D8F81662B4E4794F7D373354E256FDAB4729E724C1AD997D3A7377B5C023008ACDA3EAA90263EE7DF64982BF48804768224C703AA1CA2A15B6DD070F25103ED4 19C3101B9E0FA71EEB74FD07E91C701C58CE6B7985DA1D9F35C33F25F9BDC4D3E66C377C5D157D17E2B8E89F8347F32CC78E3EA864923B936FD7F42CDA2021F0 3495CBBF17F22790BE9B4F90C885F1F6A4FC85133C2E6BE1DA787165F93BF22FE9372F4E415ECD9ED151C2146BF93206796AD6C789529838698F6AB5CB3E22AO 7DB32F13F9F3457482CB0E61CD4EB3E8EEF7916265C254B611A2E93D808E1D0BD04153A83A96A9455E5517C3390712EE96E7EE7915A4B033CE282711324350F0 4E3EBE0A10ED7262D7A15A4CDBCB39941E05E60CF286C93ADDF3FDA7C0490AA52F4B99283B2744CB5A52D26702CFA267F0C3319B33CD621D81A7E15EBEF57300 5830A26FA319C1304BC235C691F017588214C89CC874875BADD4A34CD8CE68F3A2E5006F54BBE0740AD5651360B9C6166EBD9C903CE0485C907D167660E065F5 $3 A 334 \mathrm{D} C \mathrm{CE} 334 \mathrm{C}$ $3 \mathrm{~A} 3$ (1)

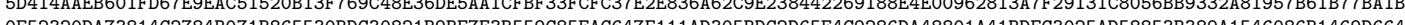
(1) $45 \mathrm{C}$ CF49DDE748 33B3D8EC8F75228AF2177EE908EDFCCD97AAE1C21B10179DD5CE8E23C4D294199A96E24317ADEB5279D8424EA3213512AD9BB141233EAC69CEE229B9BA042323 B1690CF65E10D6742F160893C3A146D674BAE9A09288B32C80C82FE9E576B8AB4C5EBED8590BB89BAE45A7D3ED467429315BCEC87670822EA4055078A8514DD A2B5235F7865635D16C33DC170DD9F090B9FB865ABBD7143FFF1004704D7380895F40E30AFF1F63A50BAC045117C249B4667571C9B6F43A713BDEEB1209F1600 CBE86E39926A972CEC6BB6058EE0C6C688D3C4BD488049783250E32F4263E26DD4B1A0EFBA04A57EDE9175F746F764046C29BB8D9C6793E60F01C9D999DCF77 A 832520BBCA91B7A961022A193C30E01940CB391577B5BB4A3779B4E8BC1DF8D2FE5EEF66F279E770352EFF5959EF4013D914AF3656F0F79CC9D08822E7DF42E 9DBF2EFCCE11315F5A2F64613C0ACCECD000971DC2D7580603C0277FC3A8EA08BBE0E829AF9C22F35F79137BABFE82172C2F2C1D512AC3D8DBCFA3FEEA4CB24 4E1349A2B22D5E16417A1D2D990BD6D9486A06DFE993DE38BF59EEC027410BFDE4C1C70CFB85A61B1A3C0DD231C4438FA150562455D524BA99E834790A416DAE C874312EE6C43B8C4B2ECCE1C9B719229113CEFF985D8EB1C7334FF59A4BC440E6C6B068792B7D0DA0E5B1A5DCC1EBC21B33769D95000B3B19567874A80174F 23FCEAA021EE20321E6DCA14B050F60D5AC1AB83BAC848ADDCCB3AC10527D5446F4A047A7968E0B502AB015B683CB6B0AE54183FC86C606AC85B454880D2F03 77B83A95FE769474D524770B3FAEC27369EEEF827AF4EE69ABE13C6169FEA05E51BF86571E7E0E6C6C5E6A7715ECBFCCD0DA3E632EAC2254D8ECD29D700C3658 1D9621A22D8FBF7F57C03C8F73EAB53EF403D66AF8E1F9219FFCA1DFE32C306524874AF07D8AAEB944A6A7D073A994CED2DBBB49E0A6115F2D55145352A4DAC7 16D448F2C06DE7E9B7CD71099426A665BD50648EABFEA1B6B120751F0A1D93D457A02716EC1046BEBAA8B31F174DC5799D6C1556ED81CAF8AFC22F5F68E9F3C5 $3847 D 5081715 \mathrm{C} 362336 \mathrm{C} 5280$ 作 E4FE486 5B4108C037 源 AFCAE E70846197ED7 1960BADAA9E73966C19615A4909752B5EB0288603E6F450AB0D138D2A19535ACC10A51015A9E76E15F6CCE6C7E065F5F942706A618607D10DDC8EA23FDC7C60 1A777105D338CE47F7F0BFB6D13BCDOFEAA3F1AA19044CBC7C0299880991CF4012F446411AE0B03303245D329DBDA957AF6CDD13C7FB52C002DB2613872AA2AE 6F1B8F7B2559B8A7E872161BF8F8941DF825D52BE2E9EF6FEF68BA89D386E628DA64D91431D9779D936D7D42207ECE5415E56224601B1AD505120BA94FC0645E 843E4BCD0C58D948C4A9C9B56A41CEBF2BA07677A4AE993823427CFF72D83086D225A987441DE4132D8706FC02A03FA94E3D4B5EF86COE9BD9B9F436AF1A32C F328A0E13916219D5F00D1A42E5378108F059CFDE34F3AFEFCFCB888F7B339C09D3B6CA9378067B51FDBAF48C60CC3AF6619AE9EBCA39FEBA0D75BFB1C67D7E7 06E154264DOF7FC168E875DEF67B0FE6520929628A2FA39F5E75868CB3C7C04025F351FF834717C6AC84AE427E5E4E7C12246CD72DE8AE3CB31C267E952455F4 F92C630E720569BD9B208FD6EBA7EDD3031E0C53F0E5375E565DC40373E11DD9ABD043B2AAD9942CB1C0557A2132070683E908AA85C51AC7F98045D1FBCD8148 35E5D9ADDD460F26E7FE74A812E5AE03868F01E67F87ECD807F844A24742160F316C21830D5BD247781E82F65D563BFA2A79400014157952A9CF5AEDD344E9B 386558FDABEF24EC7E78A97A9AC5C3C6BD4E5D4BED7779A57E0667537EB8F0833F9EFA3E5ADFFA056CB65C2C759CCE88B3A389AB9B79D2A887803F2ABC200F04 B22E5E5383A500BD196BE9C029EF1EB604D0DC6A0C7D2BCA1AB879C52D2C287452C263C4C401CB034F2A1DC398B921B56E27272189B8EA3F7B041CD90863C697 


\section{Appendix $\mathrm{C}$}

Truth table of the 15-variable balanced function with nonlinearity 16272, degree 11, maximum absolute autocorrelation value 248 and algebraic immunity 8 is as follows:

D1CB38B9790902B1F3E0735E9C7EFCDF63388A9CB6285164F57CA6DACD682BDD5FC382B3F3014F83131F3F0CBF250EFD76005C2D101EC501D36AA5E32F573B81 FBCDD382496FF996CDC3C96116761C6C34C3CE882D36899616C82BEBCC8A982FE74E32CCAED67B2A65CD9BDFC2BFD9749582B0EFBBAF302CC59D04B787A9F7DA 72AC872B2E28A380474AE48ED85A0E4E973E2C29C30EE124DE0A58F1DBC61E399207C2C6699EF6482E2A965BB71E4B1B65A47CE67E0265FD6C97F7051BF26A23 DFB2B89E6CC06DC705DA94B5A7FC6B04A401862E4EE9C5269391079DD15AA203B1FF597FACD934D813A9BE77C6763E2C7C15B52F9C56ECA3A7E740E1DDF32ABE 08C01582A6E7C1ACD49B2B581468E7CCBC0C56403506F2208158FAAF6271BDCAF4A7C2CE6F3CC4F1397C236174207AED2ACE2650BBF7DCDFC156284E657487B4 1A7F77E3686F86F15F0E5B8AC9B1F8A26E2595EFE5B0AFE903583121FDAD6146E511AEA8E3C39EE44D34997E1FBE26904F3C1B0D88E28D418EA9DC85B1AE7B87 6F98FC9502F3E5345A2D38775B2B690973FA6EEFF5AC4311411DC839B4AC67AC5056279B485F2A61162434B597EF800BA5970B2123F61BD07B600101412A3387 FDCF62DD155EB3D91592D15BD617D4088E2CAB1B468B08B2C7A1A70B6D74C0C6097D8B40B9AEC4D85AD711A025C7AE8750488EE2BD67DACF953A36293A1512DE C8F783DDCA01A2D4EEF56058D6DB108380ED0ABC3B12BBA68F460E0C751C867C4697326DBB5B57DC7DAA994B8D908522A69AFAA2CD0405C8F53A088F53C4D701 D9FC40499790298EF0982D7C7C13998F82A44D2DD46C0ECF19F9D4775C5425843E4168DA6BE1BF2706AC9DF32E83C0667D3511F1C0A312644ABFB612F6E24747 7100A68D0CA76539E5B74B231C5E998FAAD83320EFE8A341970F5230D848047E4E708444A1FB31893055F88850DD9F0B8C280109D7656E8ECD7E00945ADEE94 340121DA559EBA4DCBD36D79F0203046527E9246A55EA69E9BD8FD70845BF1D8ADD9286CDFFD232EB249347EB67BA831A72544A0917909415331BE25B2EDB6A3 B59DF44C76D3F0AB27D5333C9EAF87471050D195681CB31DEBF82B43E5A5761AA668D911F18BCE73D9EB458716978B75538BCFC1C748D2E11603EB3CB049FA7D 148DAB0B2FE65EACF8A75522COEF1B9ADE1F3EFCD346ACFAF5E375C8A698D9EDBAAF4A096652C030D12D88A58EBCC1CCE3EC1B9D8C21229B07CFD4BE29C74919 8D6A7DC94BC02AC59F0151600779854F9E11A4D13F200057C5F5DF4D90BD88E2F6658092BE039FB9E90AE6028CB3F8D1380DAECE011973131A6EE20386C9390A 8CE5093F48A9529CE81B54CFC7FD7AE244016A4C24CB5432800487E7158FF7B2EB66575908CADE91F87AF10894117989A4FF87AB39E4C5E182EA31BA9A7AD471 39E6CDE6182D907A86158C31AB853F16DE2073110AC8FBA76A1E9502DA77B3D70D268B3ECDFFAC2D7D07DF3EB956444EA776FC1F23749CC6A200616C4C1A1C78 42E05B0938C1B0C042B85452AF59D56CA7DOBE114EA04642A23E4A32B7BFD47FABB40AEEC941013B2DD433E9C954C61862142C45C9A6A26254872913C0E2DD75 42E05B0938C1B0C042B85452AF59D56CA7D2BE114EA04642A23E4A32B7BFD47FABB4OAEEC941013B2DD433E9C954C61862142C45C9A6A26254872913C0E2DD75 C68E17CEBOF7C4450173435E873D04D36E405D6B12F772D818EF7C228261BA1F7C52CFEDF25998B96DD152C7191A1061710169CADF58E3A274ACED1ED5846B7 A674328C514FF22A376C44591BAE92AOCEBB1709025781398AC614250EF4B63650AAC11C832D3BDDDF7364E2FE93FC60B1A6CCCD118487766EB61318DC120874 A674328C514FF22A316C44591BAE92AOCEBB1709025781398AC614250EF4B63650AAC11C832D3BDDDF7364E2FE93FC60B1A6CCCD118487766EB61318DC120874 1721E776A2A9AC13107088347ECA2ABBBA1F02153AA173A2CBC9593A195094F888990367D8DA95F 45C4A2DEB1C4E95B145555535154EB1710EF8D51973C14C9CF6A8BEBCCDCE7EB6 45D4811AC4F60B7C846662D07FD2D09FBD85B8B429BCA69B431F094314F32D8F045C3FFFF916FB99F0DAB6F5664FE93451D7C334F8249555066A9F2E524BFE93 B9BF5AD198E9AC86A30E85822637AA53800CC0BB863D296CF07400DCF7B6ECOD20D8AA6685CA1F45E1A62468427D602A75B8C958D4B847829FFE110449E08AEF 515E1E0D6FA460CACCDE08FC9230F278814E60B946EE085C9E5AE5F74CBD0CEAEE78F743398D829B82A3F9A68584313120A4D96C9B0787821366030B33EE483F 8B53B26F14BC76072A76705F41654093CD1C144C14AED52F2C769A229AECF5156A9A652793CE897D91D7B84FEB6877BAD9AB743919FB79B3551FB54C7FF1DB2B F80514DC4204DCE0E05F11D4692328896E716A6B49E3BFEEB27042C3838B3D97DB86208220461A9418C32ECED42EF152C949D3763E17AC757F4B2D5C461F9AB1 F63EE4BA596E38F1FDE886CCC1BB92EF0F8C768EEB250B8936E3E7A5E6EBBC8DE5609A61EFEC6E64C7DD6BCCC97715E22804C8BE3F88536EB58944294E75D602E 660F4BFE1927E480B970892DE52712F08F0ADDEE9AF19D2F19D6EDC777F7D48ED6B7627B05DEC22427CBDEE55C96DB697011F59FCB848C3D0C1BC35E83A53741 5997CEAE99E46872071D40A414C10FD39E598BB804ED3899681DDC3EA2BB9B81E80637DBB5BB126D2AE96DFC41BD927FB2899802F6B624498F55B332261DA8D1 8B57B5F14B3FFE18DB6241 8B51B5F14B3 7C1 EE425713661574CB79F157CFCD2038179490D5ACC73DDESB24BBB0682BACAF65EDLD072E78BD4578B0FBA1146B1BD0BA42F7F695F6186928D216ED13BE214 D5BADBABOAD6481BAE5CF1086087AC8BFDEE075B5503916A819A1762AEA680547BD3D1489E9BBFF65816B08EF8B54B68CCD63C2978D87B2FADF8DBEF223E4DE1 757986EFED3434F656F5E2BCD69E6D740970B80B156595D47AB4A1071B5C6BC03BB9ACEE38E765106B109B7C0659777967AFA5B7EC34C85F96FAF8438FC611C4 2D4FB7649A0CC661E98D230DC6BCE94172708B087CE9C24574E31D933B33136AC64B9FB89955D1F1BDCB80895CA28181E6DB09DE89A67C3FC0F27BA09EBCBECF 001F19E9827FB62C458FAD89DCD595FA68B3E58656ACFBF09BBDBB305A3FFEC92D8BEC12DE49675F66C0FA0DA3DED2B5B3503C4F64FCC401DC8EC8AD464F2DA5 51C851FFD38A8E9649397F3F83E9C16B3ECD08C3B0468FD58D215F289D38E036F8DF150E823D2891CF64F6A9485D58F4A18BA9F4CA287048D13C9A7E6208E602 74E78165DFF7F71A002BAA4F50C674C39A3349FA58F240B2F7F1F764FCF1BEABD4E4A47F15AA77C9B25ABB960149633EF802E51DD4FCA3BFCDC9D64BF43D57B2 436C4D5F1B648AD17CD04DBEA41C56451F54FFC7355220F876508303453C1FBCE4B1F4BB8FD6599DD61CB37FCDBC379674DA8C7069350ECC7ACC672572D79340 F297EC7848957DA858207DBF6B5453F9A9509D21C88DD525AE39BE15DB083D5184C6E82D0B54E196BFDF0F54361AFD8DF65C09A0A488970FA780F66A00B40022 47EE3C46AB953EDF1330C7A974E51052DE1600BAFA113DAE74BFF88FADDFA774663427B2D5FBCBA4F72CC3DFE9D3B42D775AAF1C138FB879C37B3D8A5C64021F 43FE7F17A28FCFEE6847C9B4CCE90C391D131FF0E53FC5DD58232A7FD4DC1AD8F93B0905F949161695A0E829041EA547BD2864279CB1438360AD377D7F3B49DC 8D07002992D8CC67C84E8E1FDD435FF4D8D82FFEACE43215E41A437375F17DA8BA25C0A76C2C38F9D94C9503A0FEEB5123E0B0BBE03294DAAB3B0247678FA5D7 428707370

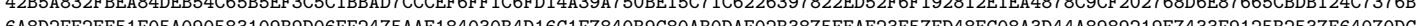
6A8D

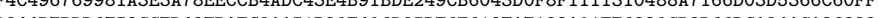
(1) 作 4E415AAESBAE904C1F3952158A0483B4F75D5A316F343339A12B4B21CB342AEC01A00A849EC83B1DBA1D956B6B4ECD3FF4064724305132C49763270901695FD 9D09DD5565AD3B87B92B26A97FC28AD870264433DA64C1A94B4A85296A4D5CA51F30B2350E14B1E9BCA19FD742999A64036E58C8A83AC7C6A69309E8382A4F5D 3BADA6E0D133F3802FD9ED1A64554B1670497C3C2A247C9E2A063F34262550C469A4160E2F7D5CA469608F1DFE38A7551926F8E87F2BABC7BD1F21364FAEE99A DDA1828BDFED13BFEAF04A3182F69CDE32809A19481B8379839A46F9F4D071DC2662089649B10D8A75AC7BBECCA8B35B965F9BD2351DFABBDBB434F8E86D0D94 74454A885EBAOA9B9A2731D24F34F44397F32BC00F473C8269F008A6CE45CFD5C5E733BC3DF7F965786648E350D2B5CFB9EF59F447854F 16745C443EB1277B21 A45E3327ADE89820340E900D8FB81873A95BE633F71EC58425552FA3CF6E8C990C9877102FC49A20E5543730E13ECOD8215A3F4F8B7B3C08A209C9F73B16E325 D4A516090B84A2A9980090343D6B2EB9D11D83033BDADFEF406D5E1F8042E5E5098D8F7598EE0ACC6CBCA7BC58D3796FC8C765473D6D4165F79254412DB3E831 545B5E0DD62054CB1AE0DFB8B1F749848D5C306AA873FAF835F92D6073362E6CB125BEA0E60613B3D8429E3AC6A7F11AE1F3257E6FD36921FE8416ECB4030974 74F662E5C7199117B8FF53E86594E0807E5160E51B2E33D6B921B83A5B3A0C36463BB75B1AE323808DB4065E2EBDADE1B665EF50FC93977ED11E213E51B390DF 48B945B31251053764ED5769D8627370F63A070F92C1F971C79BE7344730C444B42EFFF2EA8213DA48D1F7040BE18E83FFD813B593748263CEE640248CB38FEA DF1017920EA36D864169EC87EF13EE0F3819DAF928A8BC7B590DFB53F0277F9D512E84E8C306EF061F685AC8768766AD777237E8226DB906A6DD51F78A85A674 